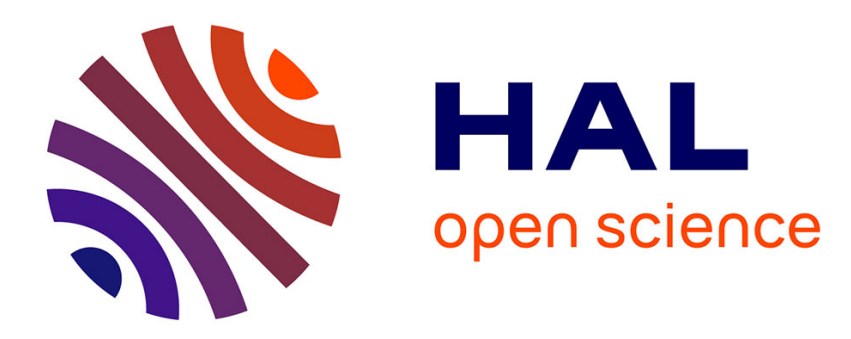

\title{
Analysis and Interpretation of Graphical Documents
}

\author{
Bart Lamiroy, Jean-Marc Ogier
}

\section{To cite this version:}

Bart Lamiroy, Jean-Marc Ogier. Analysis and Interpretation of Graphical Documents. David Doermann and Karl Tombre. Handbook of Document Image Processing and Recognition, Springer, 2014, 978-0-85729-858-4. hal-00943058

\section{HAL Id: hal-00943058 \\ https://hal.inria.fr/hal-00943058}

Submitted on 6 Feb 2014

HAL is a multi-disciplinary open access archive for the deposit and dissemination of scientific research documents, whether they are published or not. The documents may come from teaching and research institutions in France or abroad, or from public or private research centers.
L'archive ouverte pluridisciplinaire HAL, est destinée au dépôt et à la diffusion de documents scientifiques de niveau recherche, publiés ou non, émanant des établissements d'enseignement et de recherche français ou étrangers, des laboratoires publics ou privés. 


\title{
5.3 - Analysis and Interpretation of Graphical Documents
}

\author{
Bart Lamiroy and Jean-Marc Ogier
}

\section{Short introductory text}

This chapter is dedicated to the analysis and the interpretation of graphical documents, and as such, builds upon many of the topics covered in other parts of this handbook. It will therefore not focus on any of the technical issues related to graphical documents, such as low level filtering and binarization, primitive extraction and vectorization as developed in Chapters 2.1 and 5.1 or symbol recognition, for instance, as developed in Chapter 5.2. These tools are put in a broader framework and threaded together in complex pipelines to solve interpretation questions. This chapter provides an overview of how analysis strategies have contributed to constructing these pipelines, how specific domain knowledge is integrated in these analyses, and which interpretation contexts have been contributed to successful approaches.

\section{General Overview}

\subsection{Graphic Document Analysis}

Graphic Documents are basically documents containing a significant (if not exclusive) part of line drawings. They are usually considered a separate class between full text documents and photorealistic documents. Many applications or research areas related to the interpretation of documents in general, usually consider it good practice to segment composite documents into at least text, line drawings and photo-realistic sub-parts, to which more specialized treatments are then applied.

There exists a very rich literature on projects and systems related to graphical document interpretation. Some of them are inspired from concepts which were developed in broader image interpretation problems. From a conceptual point of view, the scientific objectives of these systems consist in trying to implement generic and adaptable strategies, based on knowledge modeling, adaptive interpretation scenarios, etc. Most of the systems are related to projects within organizations managing huge amounts of graphical documents, and for which these documents are operational or organizational assets the value of which needs to be optimized. Among the organizations / research groups who have presented some realistic systems, it is noteworthy to mention those projects related to the management of network maps and data, such as telephone networks, electric power grids, or waste water networks, for instance. Since efficiently operating these networks raises many management questions and is a major financial issue, many companies have investigated ways of leveraging the use their digital maps with automated tools that are capable of extracting relevant interpretations.

While there are many references in the state-of-the-art literature to various domains, applications and categories of documents, one of the most successful approaches concerns the area of electronic diagram interpretation for aircraft, done by Baum et al. at Boeing in the late 1990's. Their goal was to scan paper versions of wiring diagrams and other engineering drawings in order to convert them into a digital and operational maintenance tool, that would allow hyper-referenced access to assembly parts, or logic diagrams, etc. The illustrations below were reported in [Baum et al. 1997] and subsequent publications and give an idea of how it was able to cross-reference, text based part numbers and graphical entities. 

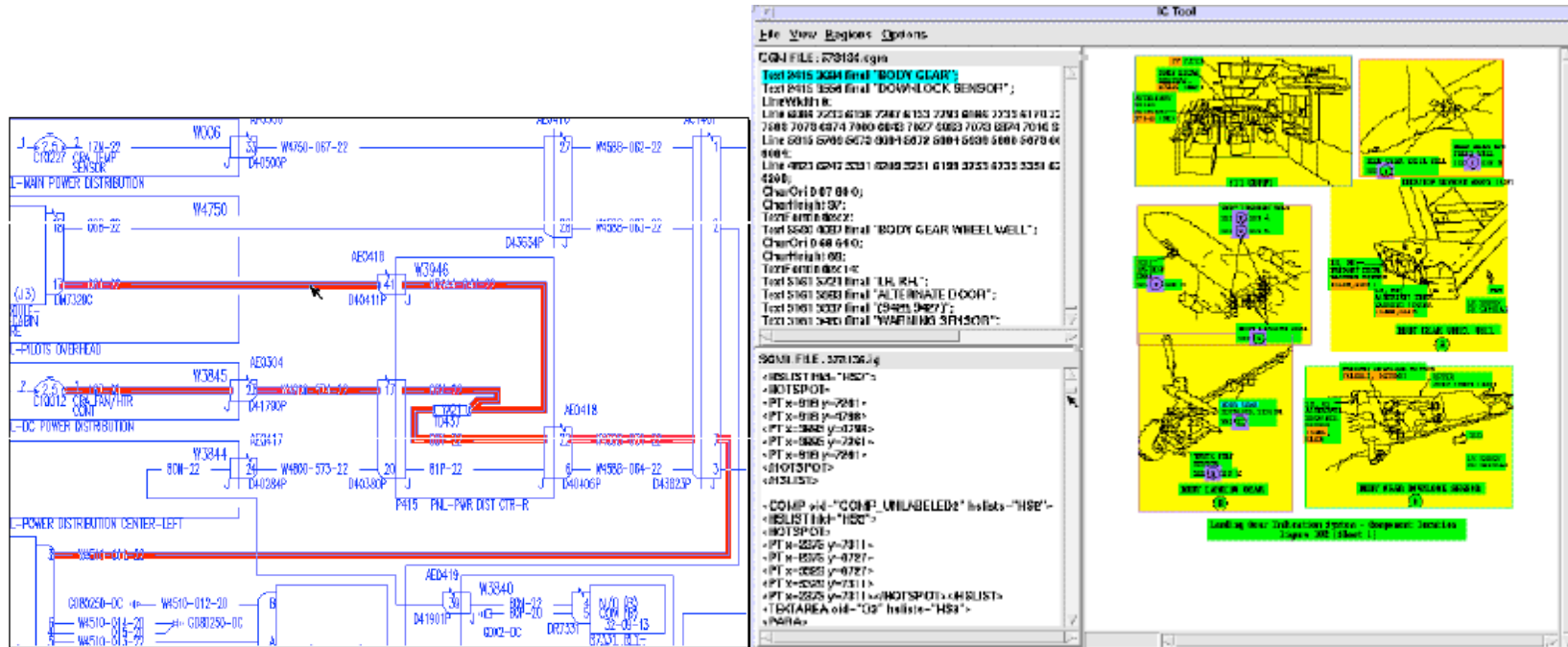

Figure 1 illustrations from [Baum et al. 1997] illustrating the results of graphical analysis tools linking textual and graphical data to aircraft maintenance semantic information.

The main challenge of interpretation systems is to implement flexible and adaptive strategies, being able to produce knowledge compatible with its interpretation domain, and being able to automatically detect and solve semantic ambiguities.

\subsection{History - Evolution of the problem}

The history of automated document analysis and its related problems finds its origin and initial scope in the corporate business domain. In order to optimize information management in big public or private institutions which was initially totally paper based, new solutions and document management applications were expected to emerge from focused research. In this context, during the last decades, mainly due to the huge increase and reduction of costs of storage capacities, and to the continuous progress in image analysis techniques, there has been a considerable evolution in the various strategies of large institutions when dealing with document analysis problems.

Initially, because of the lack of relevant automatic interpretation systems, most of the organizations decided to only digitize their documents, in order to obtain a representation of data (and therefore, mistakenly, information) which could be easily stored, shared, duplicated and transmitted and would therefore respond to elementary data management problems. This massive digitizing cannot be considered as part of document analysis per se, but it marks the beginning of an era where automatic document management will progressively attract more and more economic interest. 


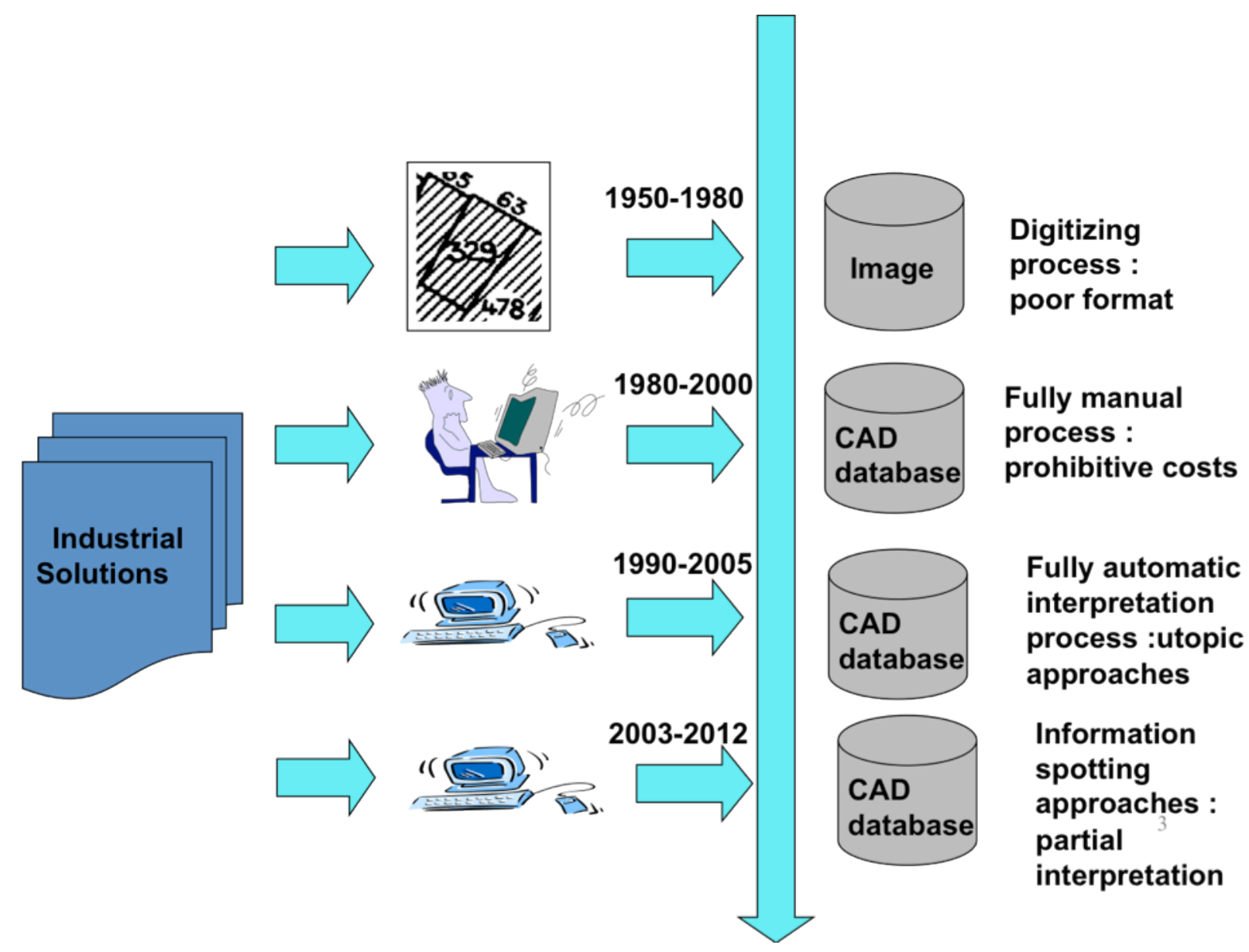

Figure 2 : Main periods of the graphic interpretation history

However, it became rapidly clear that considering digitization only was producing data with too poor a level of information, and most organizations decided it was necessary to improve the level of usability by trying to obtain higher level interpretations from the digitized data by structuring the contained information through analysis processes. At first, regarding the fact that automatic processes were in their infancy, most of companies and organizations adopted full manual processes for reverse document interpretation. Many low cost manual digitizing and interpretation projects were started in countries where human labor was cheap. With respect to the definitions and classifications mentioned in the previous section, this is an interesting benchmark, since it fully relies on human document analysis. It remains interesting to have a deeper look into the notions of context, interpretation and analysis in this particular case. Indeed, the analysis is done by human collaborators who are not necessarily entirely knowledgeable of the interpretation context, since they are externally hired to process the documents and provide the interpretation. This required the context to be documented, and a quality process to be instituted in order to make sure that the produced interpretations were conforming to the expected context. However, facing prohibitive costs of the manual interpretation resources, and considering quality problems related to human digitizing, many of them tried to implement interactive processes, coupling reliable image processing tools and human correction interfaces. Then, during the 1990's full automatic interpretation systems were presented in the literature, based on complex approaches, integrating sophisticated strategies. The complexity of these approaches essentially came from the inability of the developers to fully capture the interpretation context, who therefore resorted to compensating this (often unconsciously) by embedding them into the algorithms themselves. Because of this, the produced analysis programs were often very focused on specific interpretation contexts, without 
offering any satisfactory hints to whether they could be adapted to other contexts without significant re-engineering. Considering that fully automatic systems that would also be generic and usable over a wide range of interpretation context were considered as quite Utopian by some authors, and given the fact that this seemed to be confirmed by the observation of the state-of-theart, there a significant paradigm shift appeared suggesting to develop alternatives to full interpretation systems by only partially interpreting document contents and by using "spotting techniques" integrating "contextual information". Generally speaking, the Information Spotting problem can be defined as the location of a set of regions of interest from a document image which are likely to contain an instance of a certain queried object without explicitly recognizing it. The most famous applications of this kind of concepts are word spotting on manuscript documents on the one hand, and symbol spotting in the context of graphic documents on the other hand. One of the main applications for information spotting methods is its use in large collections of documents. In a sense, this is a very pragmatic answer to the previously mentioned inability to capture interpretation context. Spotting techniques implicitly admit that full interpretation seems to be out of reach, and focuses either on very generic ("low level") analyses, either on very broad interpretation contexts, leaving it to a later stage process step to combine this partially complete information to achieve full interpretation.

This explains why more recent literature does much less focus on complete interpretation systems. Figure 2 illustrates these different alternatives, as well as corresponding milestones during the last 5 decades.

\subsection{Structure of this Chapter}

As will become gradually clear through the development of this chapter, there is no precise or generally adopted approach to Graphical Document Analysis. Furthermore, there is a significant overlap with the more general Machine Perception domain, and sometimes we may digress to illustrate some approaches that are appropriate in that area.

However, it is possible to identify broad categories of approaches to graphical document analysis. We shall highlight those in section 2 . Section 3 will give a quick state-of-the-art overview of some of the most significant approaches for each category.

\section{State-of-the-Art and Classification of Graphical Document Analysis}

\subsection{Overview of graphic document analysis problems}

The most generally adopted approach to graphic document analysis is related the document reverse production process. This essentially means that the analysis aims at extracting information from a $2 \mathrm{D}$ representation. The principle of document production and the corresponding reverse interpretation is represented in Figure 1.

As a consequence, a classical analysis process includes several stages which try to reconstruct the higher level information from a 2D representation, on the basis of a progressive analysis, going from low level information (i.e. graphic primitives) to a conceptual (semantic) level. Figure 1. gives an overview of this analysis process. The upper part of the figure (in red) describes the production process where an author within the context of her own conceptual schema will express her ideas using a logic (or otherwise formalized) representation language. This expression will then be transcribed and made physically available (as pixels, ink strokes, etc.) 
Object Formalism : Caption

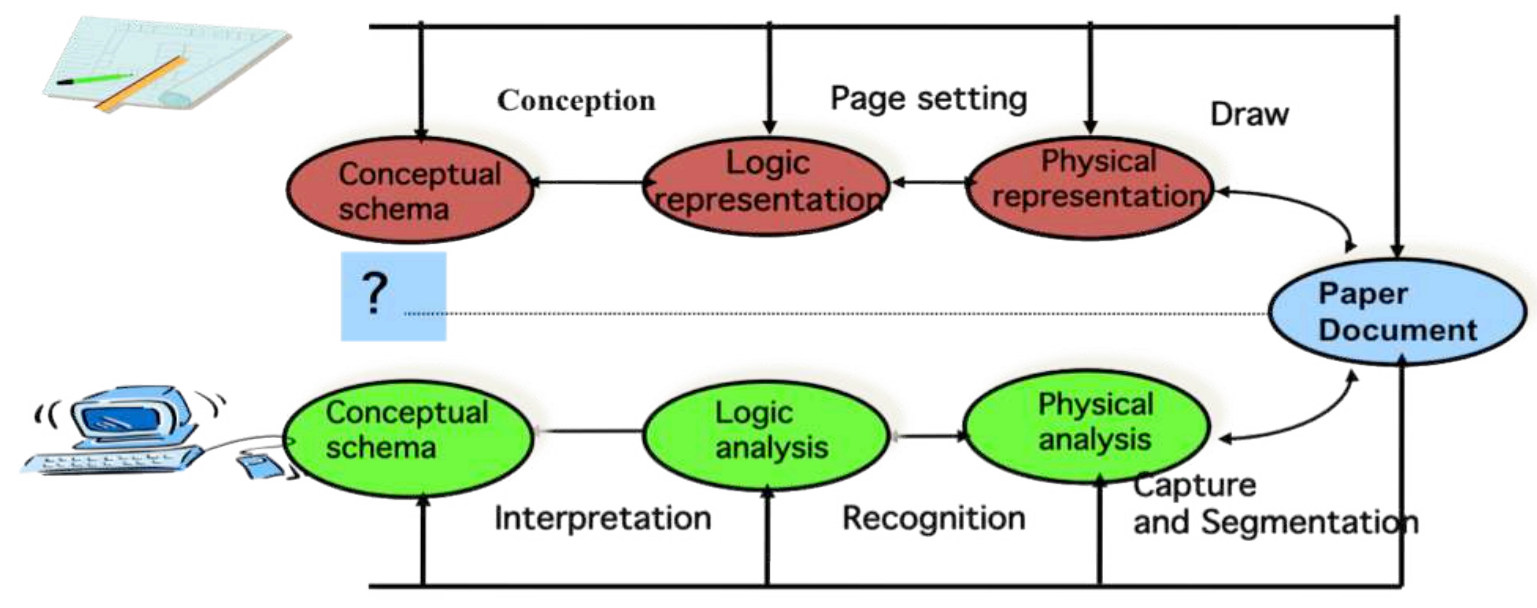

INTERPRETATE

Document Interpretation Process

Figure 3 From the production of the document to its interpretation

Interpretation of the initial intentions of the author can only be achieved through the appropriate reverse analysis (in green, at the bottom of Figure 3.) provided that each process stage shares enough of the initial context to analyze the data. Consequently, the implementation of an analysis system requires the sequencing of many processes allowing to reach this goal and covering all the aspects required for reaching a semantic level of information, usually starting from a $2 \mathrm{D}$ digital image. The way these processes interact, and the choice of which process to execute next, as well as the operational parameters they are fed, heavily depends on the interpretation context. Many of these processes include low level image processing techniques, primitive extractions, structural and statistical pattern recognition techniques, and semantic analysis. Each of these processes have already been described in great detail in the various chapters of this handbook.

The semantic analysis is principally driven by the knowledge related to the representation of symbolic objects on documents, which, in its turn, drives the different processes, tunes their parameters, stores the progressively extracted knowledge, and performs specific processing when semantic ambiguities are detected.

The main differences between the existing contributions related to analysis systems come from the way processes are organized and knowledge (and context) are (or are not) managed, from how semantic constraints are handled and how users are integrated in the analysis loop to achieve the required interpretation. The state-of-the art literature contains a large variety of alternatives addressing these issues. Part of them are detailed in section 3. The next section tries to establish a classification of the available approaches.

A special mention should be made for hand sketched graphics recognition, some techniques of which are developed in chapter 7.3 of this handbook. They generally apply to dynamic, on-line recognition contexts. A part from some very recent references such as [Mas 2010] and [Broelemann 2012] this field of research only rarely incorporates complex interpretation goals. Most of the references of this research area deal with recognition issues and rarely consider interpretation strategies as those developed here.

\subsection{Classification of Graphical Document Analysis Systems}


In order to get a structured view of the existing approaches to Graphical Document analysis, we have taken four different axis of observation ${ }^{1}$ :

1. The application domain: what kind of documents is concerned?

2. What specific visual representation context characterizes the documents and what features are appropriate to the problem? In some cases the set of features is fixed, in other cases, the authors propose training capabilities to the system, in order to give them the ability to learn from examples and adapt to different feature sets.

3. How is knowledge is modeled? Knowledge modeling is often tightly related to how the visual representation context and features are chosen (it may, for instance, depend on the structural / statistical description of the objects). Some authors try to differentiate the knowledge representation formalism from the one which is used for the object recognition process as is the case for those using ontologies [Raveaux 2010] semantic networks [Antoine 1992] or constraints networks [Pasternak 1995]. Knowledge can also be distinguished in the way authors store it, according to the reasoning process which is used in the interpretation module.

4. How is the interpretation strategy implemented or guided? The interpretation strategy can be completely hard-wired in the case of bottom-up approaches, but it can also be guided by a blackboard in the context of centralized reasoning process [Vaxivière 1995], it can be distributed in multi-agent systems [Ogier 2001Grenier 2000, Arias 1995]. Furthermore, the reasoning process handling the interpretation strategy can either be implemented as predefined interpretation scenarii (which often rely on bottom up strategies), or, on the other hand, rely on opportunistic approaches based on progressive assessment of the knowledge which is produced by the different modules of the system.

The following table gives an overview of how the previous criteria apply to the main systems documented in the literature over the last couple of decades. It has to be noted that besides giving an overview of how interpretation strategies have been implemented, it is quite impossible to proceed to a more thorough objective analysis of these approaches and establish quality measures or possible rankings. This is due to the fact that, unlike what has occurred for more classical pattern recognition problems (symbol recognition, signature relevance analysis, ...) or low level processing evaluation (binarization, segmentation, ...), the document analysis community has never organized interpretation evaluation contests. On the hand, this lack of objective evaluation is due to the difficulty of implementing comparison of semantics on the basis on numerical scoring, and on the other hand it is due to the fact that many research teams abandoned this exhaustive interpretation objective in favor of spotting information problems.

However, it is possible to provide some qualitative aspects guiding the reader in the implementation of an interpretation system. These qualitative aspects concern the facility of implementation, as well as the ability of the system to incrementally integrate new knowledge, and to automatically analyze the semantic consistency to the interpreted data, compared the expectations of the user.

When dealing with an interpretation problem, the first point that must be considered in the implementation of a complete system is the necessity to simply formalize and externalize domain knowledge, in order to define the expectations of the user in terms of interpreted objects. This formalization must be based on user friendly interfaces allowing the user to define which objects she is expecting for and how they are graphically represented. From this point of view, the most relevant approaches are the one based on ontologies, that permit to simply express this kind of knowledge [Raveaux 2011].

\footnotetext{
${ }^{1}$ There are many possible strategies to address the general interpretation problem, and categorization is always a fairly arbitrary task.
} 
Considering the different manners to store the information, based on a centralized (mainly based on blackboard principle) vs. more distributed approaches (often multi-agent and multi-operator based), centralized knowledge based systems seem to be much easier to implement, compared to distributed approaches, for which it is quite difficult to maintain overall consistency.

Concerning the interpretation strategy, while bottom-up and planified approaches were the most widely developed at the beginning of these research studies, cyclic approaches [Ogier 2000, Joseph 1992] offer some very interesting advantages related to the possibility of using opportunistic interpretation strategies, often based on automated semantic consistency analysis.

At last, the interpretation process often relies on the use of pattern recognition tools, which can either be based on statistical description of objects, or on structural description. From this point, even if the statistics based approaches appear to be much more interesting from the algorithmic point of view, their poorness of description gives a large advantage to structural based approaches [Pasternak 1995].

At last let's mention recent approaches, which consider user interactions allowing the system to integrate corrections provided by the user in the interpretation process, and sometime providing the possibility to infer on the knowledge of the system [Raveaux 2011, Ogier 2001]

\begin{tabular}{|c|c|c|c|c|}
\hline $\begin{array}{l}\text { Application } \\
\text { domain }\end{array}$ & $\begin{array}{l}\text { Features of } \\
\text { handled } \\
\text { documents }\end{array}$ & References & $\begin{array}{l}\text { Features of the systems } \\
\text { Knowledge modeling }\end{array}$ & Interpretation strategy \\
\hline $\begin{array}{l}\text { engineering } \\
\text { drawings, and } \\
\text { mechanic charts }\end{array}$ & $\begin{array}{l}\text { Black and white, } \\
\text { containing lines, } \\
\text { symbols, and } \\
\text { texts. } \\
\text { Existing captions }\end{array}$ & $\mid \begin{array}{l}{\left[\begin{array}{l}\text { Joseph } \\
1992] \\
\text { [Vaxivière } \\
1995] \\
\text { [Pasternak } \\
1995]\end{array}\right.} \\
{[\text { Dori 1999] }} \\
\text { [Das 1997] }\end{array}$ & $\begin{array}{l}\text { Blackboard, modeling of } \\
\text { Knowledge based on } \\
\text { grammars } \\
\text { Blackboard based } \\
\text { modeling } \\
\text { Declarative language } \\
\text { based knowledge } \\
\text { modeling, blackboard } \\
\text { Object based approach, } \\
\text { hierarchical class } \\
\text { decompositions, } \\
\text { Object based approach } \\
\text { defining the expected } \\
\text { properties of objects }\end{array}$ & $\begin{array}{l}\text { Cycle of perception, opportunistic } \\
\text { strategy, Perceptive cycles, Neisser } \\
\text { perceptive principles } \\
\text { Planified bottom up approach } \\
\text { Bottom up approach } \\
\text { hypothesis emission/validation } \\
\text { principle, dynamic planification } \\
\text { Bottom up approach for interpretation; } \\
\text { decomposition of the interpretation in } 5 \\
\text { specialized subsystems specialized for } \\
3 \text { D object reconstruction }\end{array}$ \\
\hline $\begin{array}{l}\text { telephone } \\
\text { networks, electric } \\
\text { wiring diagrams, } \\
\text { gas distribution } \\
\text { network plans }\end{array}$ & $\begin{array}{l}\text { Black and white, } \\
\text { containing lines, } \\
\text { symbols, and } \\
\text { texts. } \\
\text { Existing captions }\end{array}$ & $\begin{array}{l}{[\text { Arias 1995], }} \\
\\
{[\text { Den Hartog }} \\
1996]\end{array}$ & $\begin{array}{l}\text { Blackboard, heuristic } \\
\text { based knowledge } \\
\text { introduced in the source } \\
\text { code } \\
\text { Adaptive strategies based } \\
\text { on results assessment, } \\
\text { perceptive cycles, } \\
\text { semantic network for } \\
\text { knowledge modeling }\end{array}$ & $\begin{array}{l}\text { Multi operator based approach based on } \\
\text { cooperation between low level } \\
\text { operators, emission/validation strategies } \\
\text { Centralized control,opportunistic } \\
\text { strategy }\end{array}$ \\
\hline electronic diagrams & $\begin{array}{l}\text { Black and white, } \\
\text { containing lines, } \\
\text { symbols, and } \\
\text { texts. } \\
\text { Existing captions }\end{array}$ & [Yu 1997] & $\begin{array}{l}\text { Blackboard, heuristic } \\
\text { knowledge introduced in } \\
\text { the source code (no } \\
\text { externalization) }\end{array}$ & Bottom up and planified strategy \\
\hline geographic maps & $\begin{array}{l}\text { Coloured } \\
\text { documents, } \\
\text { containing } \\
\text { textures, lines, }\end{array}$ & $\begin{array}{l}\text { [Smeulders } \\
1998] \\
\text { [Shimada }\end{array}$ & $\begin{array}{l}\text { Structure based approach } \\
\text { for knowledge modeling } \\
\text { Distributed knowledge on }\end{array}$ & $\begin{array}{l}\text { Rule based strategy and process } \\
\text { assessment strategy } \\
\text { Distributed approach, Agent based }\end{array}$ \\
\hline
\end{tabular}




\begin{tabular}{|c|c|c|c|c|}
\hline & $\begin{array}{l}\text { symbols, and } \\
\text { texts. } \\
\text { Existing captions }\end{array}$ & $\begin{array}{l}\text { 1995] } \\
\text { [Samet } \\
1994], \\
\\
\text { [Boatto } \\
\text { 1992] } \\
\text { [Tanaka } \\
1993]\end{array}$ & $\begin{array}{l}\text { agents and blackboard for } \\
\text { centralized information } \\
\text { Blackboard approach, } \\
\text { heuristic knowledge } \\
\text { introduced in the source } \\
\text { code } \\
\text { Blackboard, Graph based } \\
\text { knowledge modeling } \\
\text { Blackboard, Structural } \\
\text { knowledge representation }\end{array}$ & $\begin{array}{l}\text { approaches } \\
\text { User interaction based interpretation } \\
\text { strategy } \\
\text { Rule based approach, } \\
\text { First phase : low level planification } \\
\text { based approach; second phase : adaptive } \\
\text { strategy based on production rules, third } \\
\text { phase : interactive interface for adaptive } \\
\text { strategy, and incremental learning }\end{array}$ \\
\hline cadastral maps & $\begin{array}{l}\text { Black and white, } \\
\text { containing lines, } \\
\text { symbols, and } \\
\text { texts. } \\
\text { Existing captions } \\
\text { - high precision } \\
\text { of document }\end{array}$ & \begin{tabular}{|l|} 
[Suzuki \\
$1990]$, \\
[Antoine \\
$1992]$, \\
[Janssen \\
$1995]$, \\
[Ogier 2000] \\
\\
[Raveaux \\
2010-a]
\end{tabular} & \begin{tabular}{|l|}
$\begin{array}{l}\text { Blackboard, semantic } \\
\text { networks }\end{array}$ \\
Blackboard, object model \\
approach. \\
Ontology based approach \\
centralized modeling \\
system
\end{tabular} & $\begin{array}{l}\text { Bottom up approach, centralized } \\
\text { strategy } \\
\text { Cyclic interpretation strategy, according } \\
\text { to the semantic consistency of the } \\
\text { interpreted data } \\
\text { Dynamic interpretation scenario,based } \\
\text { on adaptative strategy } \\
\text { Graph based strategy }\end{array}$ \\
\hline $\begin{array}{l}\text { architectural floor } \\
\text { plans }\end{array}$ & $\begin{array}{l}\text { Black and white, } \\
\text { containing lines, } \\
\text { symbols, and } \\
\text { texts. } \\
\text { Existing captions } \\
\text { - high precision } \\
\text { of document }\end{array}$ & $\begin{array}{l}\text { [Dosch et al. } \\
2000]\end{array}$ & $\begin{array}{l}\text { Centralized knowledge, } \\
\text { structure base } \\
\text { descriptions, Knowledge } \\
\text { based on constraints } \\
\text { networks }\end{array}$ & $\begin{array}{l}\text { Progressive solution construction, on } \\
\text { the basis of the checking of primitives } \\
\text { properties in a constraints network }\end{array}$ \\
\hline $\begin{array}{l}\text { Hand sketched } \\
\text { graphics }\end{array}$ & $\begin{array}{l}\text { Black and white, } \\
\text { mansucript } \\
\text { containing text, } \\
\text { lines and symbols }\end{array}$ & $\begin{array}{l}\text { [Broelemann } \\
\text { 2012] }\end{array}$ & $\begin{array}{l}\text { heuristic knowledge, } \\
\text { Blackboard }\end{array}$ & Bottom up and planified strategy \\
\hline
\end{tabular}

Table 1 : interpretation systems and application domains.

Some of these systems try to implement generic and flexible interpretation strategies, most of the time on the basis of explicit knowledge modeling. However, they generally remain quite domain specific, due to the high number of heuristics introduced in the processing chain. In this context, the commonly accepted notion of interpretation of graphic documents is to consider it as the result of an automated analysis process converting a poor format document (paper, pdf) into a format close to human interpretation. This, of course is only partially satisfactory, since it defines computer interpretation in terms of human interpretation, without fully assessing what the latter actually entails. The rest of this chapter will develop in detail how these various approaches and applications have been constructed, and how they consider "interpretation" of graphical data.

\subsection{Relations between Machine Perception and Image Analysis Systems}

The interpretation problem is a widely spread question, especially in computer vision communities. In many cases, document interpretation strategies can be partially inspired from computer vision communities, in which many image interpretation systems were also developed. Indeed, in the last 
fifty years, a lot of image interpretation applications have been developed in many fields (medicine, geography, robotics, industrial vision ...). However, we know that image processing specialists design applications by trial errors cycles and that there is no identifiable tendency to reuse already developed solutions. The lack of application modeling and context formalization may be a reason for this behavior:

- Accounts of full analysis systems are rare. Usually, publications focus on specific parts of the analysis pipeline, highlighting the scientific and theoretical foundations of their contributions. Very often, these reports conclude by providing experimental validation on specific data, claiming an improvement over competing approaches on the same, or similar data. This results in a very result-focused definition of interpretation problems and obfuscates, in some way, both the actual interpretation context, on the one hand, and a formal description on how the analysis process advocates between possible choices.

- The reusability of these applications is therefore very poor because the limits of the solution applicability are not explicit. Moreover they often suffer from a lack of modularity and the parameters are also often tuned manually without giving explanations on the way they are set. Besides, these parameters, and their impact on the final interpretations hold a tight relationship with the interpretation context, as already stated before. If the context cannot be formalized on the one hand, and if the parameter domain cannot be mapped to the context, reusability and generality can only occur through trial and error tuning.

There exist some approaches that try to address these issues, however. Knowledge based systems such as OCAPI, MVP or BORG were developed to automatically construct image processing applications and to make explicit the knowledge used to solve such applications.

However, most a priori knowledge of the application context (sensor effects, noise type, lighting conditions, ...) and the interpretation goal to achieve were still more or less implicitly encoded in the knowledge base. This implicit knowledge restricts the range of application domains for these systems and it is one of the reasons of their failure.

More recent approaches bring more explicit modeling but they are all limited to the description of business objects for detection, segmentation, image retrieval, image annotation or recognition purposes. But they do not completely tackle the problem of the application context description and the effect of this context on the images (environment, lighting, sensor, image format). Moreover they do not define the means to describe the image content when objects are a priori unknown or unusable (e.g. in robotic, image retrieval or restoration applications). They also assume that the objectives are well known (to detect, to extract or to recognize an object with a restrictive set of constraints) and therefore they do not address their specification.

\section{Examples of Analysis Approaches and Interpretation Knowledge}

\section{Representations}

It should be clear to the reader, by now, that from a technical point of view, there is no formalized and standard approach, nor definition of graphical document analysis and interpretation. There are merely interesting and successful approaches that have proven efficient in specific application contexts. Taking a closer look to those, there are, however, some lessons to be learnt from how they integrate various levels of knowledge and what strategies are deployed to make them as flexible as possible to adapt to other contexts. This section will try and provide an overview of these strategies.

\subsection{Classical strategies : bottom-up approach}

Image or document analysis is a difficult task since it requires a large amount of different data processing techniques, from low-level treatments (e.g. noise filtering, data restoration) to high-level interpretation (e.g. object identification, decision making) through intermediary operators (e.g. segmentation). In order to solve this problem, most of the different strategies available in the literature are very much based upon the hierarchical decomposition of the problem shown in Figure 1. 
From the acquired data, treatments are most of the time run sequentially within a bottom-up strategy. Each operator of this decomposition provides a result, constituting the entry of the next operator. Following this approach, the most sensitive points are the choice of the optimal operators, the definition of the adequate sequential ordering of these treatments, the management of the quality (or uncertainty) of their results and the communication between the different levels. Most of the time, this kind of conventional approach relies on three main levels (see Figure yy), each of which manages a particular level of information:

- the first level manages the extraction of low level primitives: it often includes prepossessing techniques and extraction of primitives (lines, circles, textures, textual information, ....) The techniques and tools related to this have been described in chapter 5.1 "Graphics Recognition Techniques".

- the second level generally manages statistical, structural or syntactic information, and tries to combine low level primitives into syntactically, structurally or statistically described objects, on the basis of classification techniques, graph matching approaches, or syntactical methods. Most of the approaches related to this level have been described in chapter 5.2 "An overview of Symbol Recognition".

- The third level generally tries to integrate semantic constraints, in order to solve ambiguities. This level is usually the less formalized, and forms the core focus of this chapter.

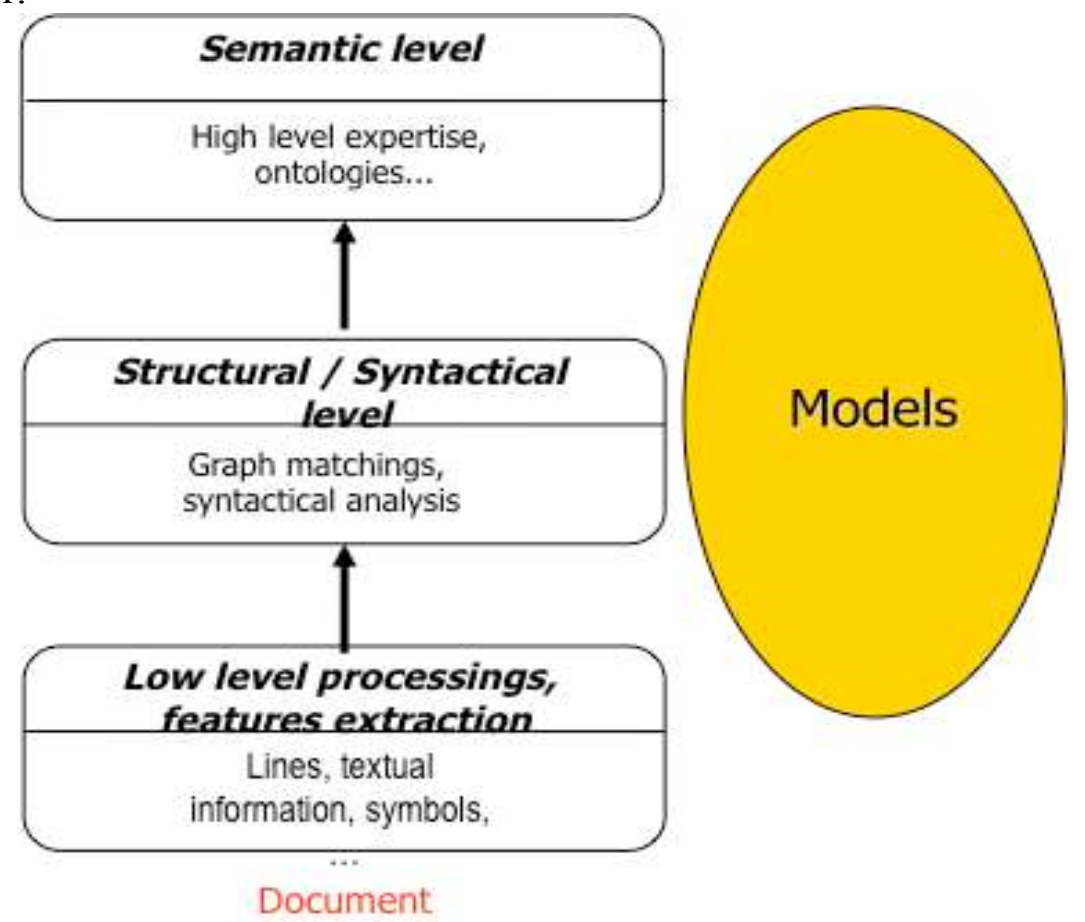

Figure 4 : illustrations from [Ogier 2000-a] illustrating the main steps classical bottom-up interpretation approach

Most often, graphical document analysis follows a bottom-up strategy. Algorithms are performed in a fixed sequence, usually starting "low-level" analysis of the gray level or black and white image (sometimes combined with noise filtering and binarization cf. appropriate chapter), in which primitives are extracted by specialized operators. Generally, these primitives correspond to segments, associated or not to polygonization algorithms, to symbols and characters, textures, circles or circular arcs, dashed lines, arrows, etc.

In the next phase, associations between all or a part of these primitives are detected, and higher level graphical entities are constructed, guided by some a priori knowledge. This knowledge is either directly written into in the source code, or it can be declarative knowledge based on explicit rules for graphical entities. An analysis of graphical entities and their relationships allows one to 
propose an interpretation, in the case of strictly bottom-up approaches such as [Boatto 1992], [Deseilligny 1998], [Kasturi 1990], [Shimotsuji 1992], [Suzuki 1990]. The main difficulty in this kind of process comes from obtaining significant and robust graphical entities from the low-level operators and reliable association rules between each primitive in order to achieve a correct interpretation. These issues have already been partially discussed in Chapter 5.1. In fact, contradictory as it may seem, these systems all extract low-level primitives the same way, using the best state-of-the art approaches as off-the-shelf tools, without necessarily taking into account the specificity of the visual representation of each graphical object within the context they are confronted with. As a consequence, due to the variability in representation and the manual finetuning of many of the intervening parameters as well as the hand made -and fixed- combination of supporting extraction and detection algorithms, many situations in technical documents are difficult to solve by these approaches. They usually concern the connection and overlapping between different visual entities (e.g., text, lines and texture), text identification in handwritten annotations, isolated character recognition under multi-orientation constraints ${ }^{2}$ (for instance in city maps, or utility maps), low image quality, and variability in the representation of graphical entities. For all such strictly bottom-up systems, the main problem is related to the poor adaptation of the parameters of the extractors and to the inadequacy of operators to the local features of certain objects.

The most emblematic bottom-up approaches in the graphics literature are [Kasturi 1990] and an updated version, applied to architectural drawings, adapted to the evolution of low level treatment and higher level recognition processes [Dosch et al. 2000]. It is interesting to note that [Kasturi 1990] considers "shapes" as the ultimate level of interpretation, regardless of what these shapes may represent. This means there is a complete lack of semantics in this approach. The goal of the approach is to have a geometrical description (vector image) that would be as faithful as possible to the initial raster image but that would go beyond strokes and connected lines, and incorporate coherent descriptions of shapes (circles, hexagons, parallelograms, etc.). [Dosch et al. 2000] extend this low-level consideration to not only integrate symbol recognition, but also adding an interpretation step that is targeted toward their application context: architectural drawings.

\footnotetext{
2 OCR in graphical documents is a different challenge than character recognition addressed in other chapters in this handbook. Textual references are very often very short sequences for which no "text-only" context is available, as in full text environments (where dictionaries, or other linguistic heuristics can guide in solving non determinism). Very often the interpretation context of textual annotations is related to the graphical context on the one hand, and syntactical reference conventions or encoding on the other hand.
} 

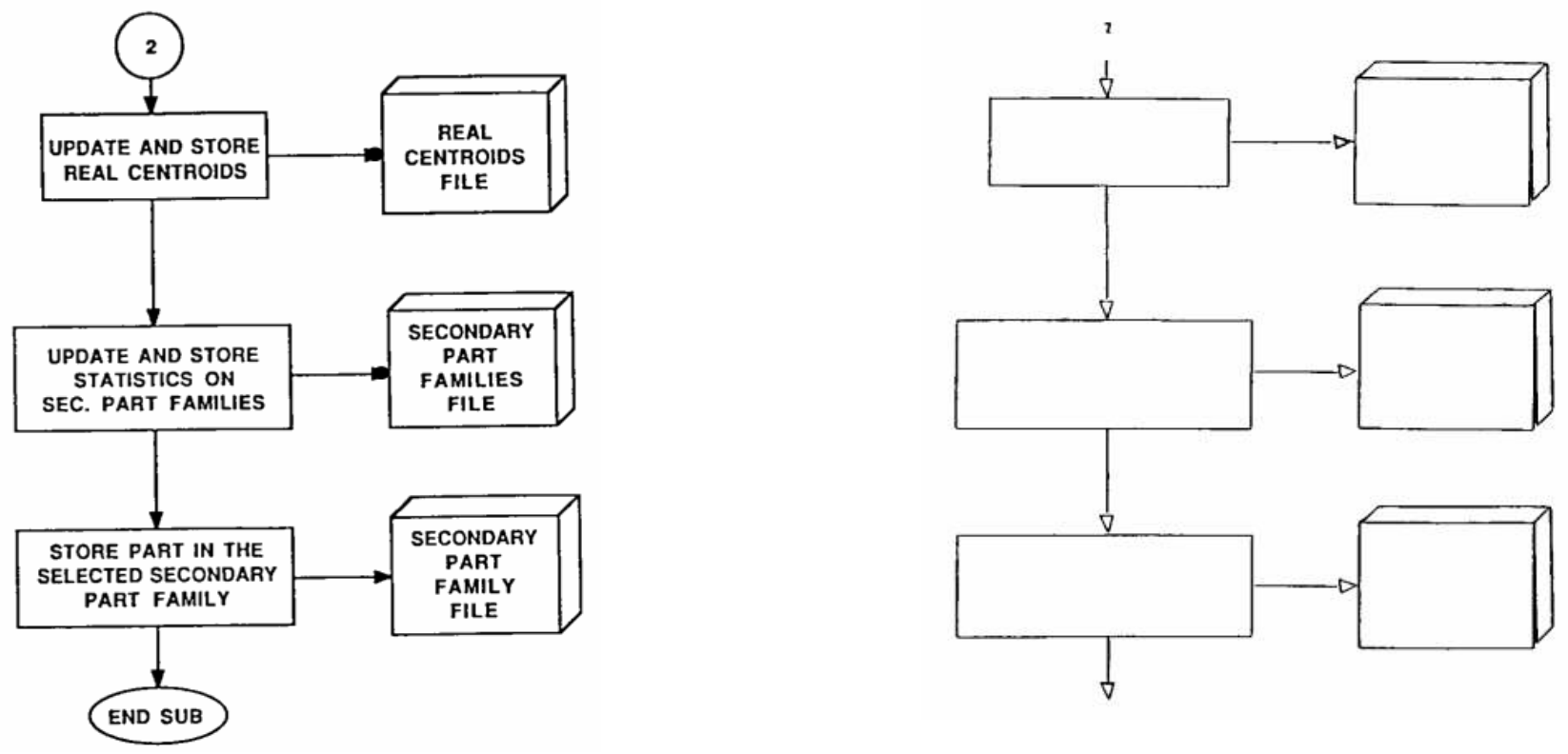

Figure 5 taken from [Kasturi 1990] and showing the interpretation result. Note a minor segmentation error identifying the upper ' 2 ' as graphics, and the vertices not overlapping for some of the 3D boxes.

Since it lacks a more semantic verification step, the former has interpretation artifacts like those shown in Figure 5. While the method (almost) correctly separates geometric shapes from text, etc. it identifies all 2D polygon shapes independently, failing to establish that they stem from a 3D projection, and thus missing the vertex co-occurrence constraints on some boxes.

In [Dosch et al. 2000] the bottom-up approach is extended to incorporate more elaborate shape recognition on the one hand, but also to relate them (and their visual context) to architectural representation knowledge, as to model $3 \mathrm{D}$ buildings from $2 \mathrm{D}$ scanned images, as shown in Figure 6.

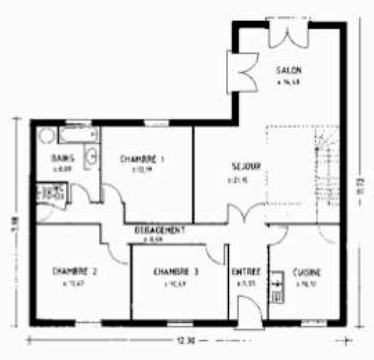

(a) A drawing.

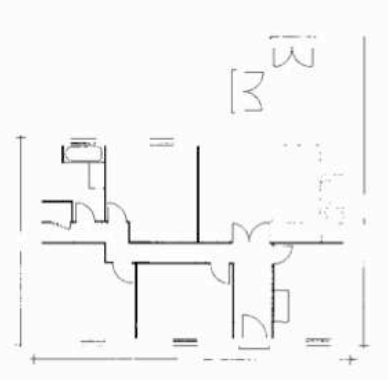

(b) Thin lines.

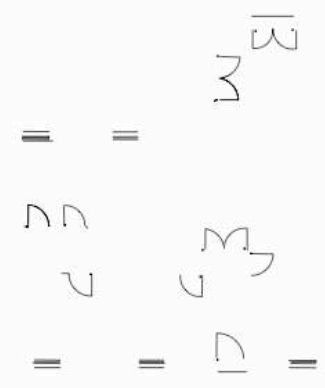

(c) Symbols.
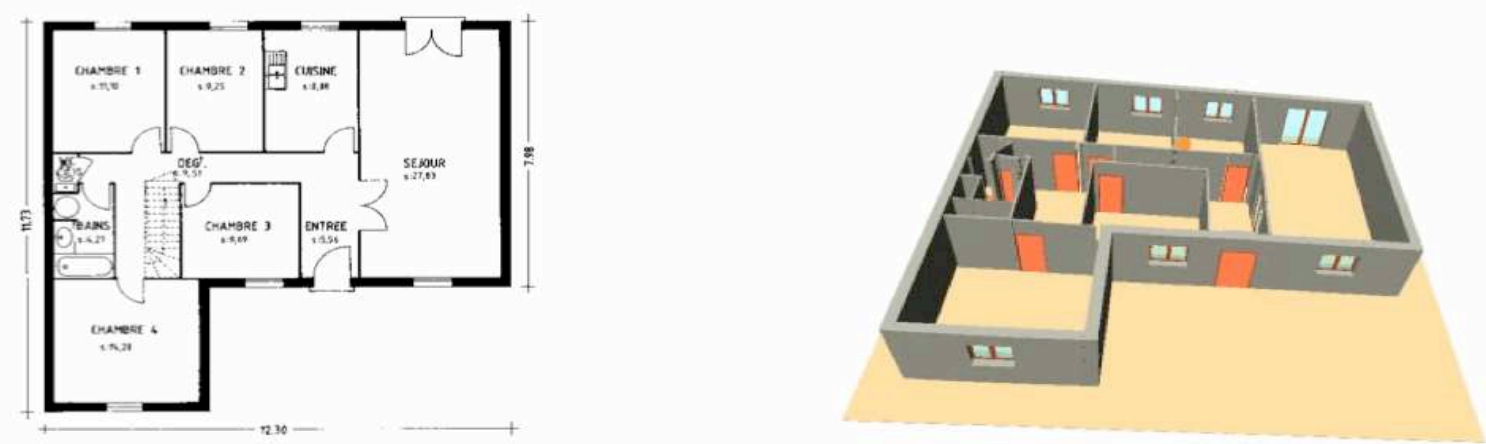

Figure 6 from [Dosch et al. 2000] representing the results at all major bottom-up stages of the interpretation process: line segmentation, symbol recognition, context identification, 3D reconstruction. 
Recent approaches have tried to revisit this paradigm in the light of symbol spotting, without fundamentally changing the three levels described before. The main shift is operated at the low level extraction where, instead of trying to extract features justified by human interpretation semantics (lines, text, textures), either non-discriminate small areas are extracted [Rusiñol 2010], at the standard image scale, scale-invariant interest points are extracted [Nguyen 2009] or patches corresponding to regions of interest are used. These are generally based on pure signal processing techniques identifying the maximum of entropy of information-like zones [Li 2012]. They have been described in Chapter 5.2, and although they have proven to be quite efficient in lab environments [Coustaty 2012], they have never really been integrated in graphical document analysis contexts beyond symbol recognition. One of the current main obstacles to mainstream development of these approaches in broader analysis processes comes from the fact that there currently is no trivial approach to integrate signal-based patches with the higher levels handling syntactic and semantic constraints.

As a summary, one could say that the major drawbacks of the bottom-up approaches are due to the fact that the processes running at each of the cited levels do not sufficiently integrate contextual information, if any. At the lower level, image processing techniques and features extraction are run globally on the whole image, without integrating local contextual knowledge. This highly conditions the quality of the extracted information. These results are transferred from one level to another without many possibilities of coherence or quality verification. Another key problem of this kind of approach is related to the fact that many sources of knowledge are implicitly embedded in the interpretation process, and this knowledge usually is tightly linked to the data and to the targeted application. This drawback makes it difficult for this kind of approaches to be reused in other contexts. Furthermore, the lack of definition of a memorization strategy in order to apply the most adapted analysis sequence for a specific context, by using contextual information, but also by using the history of the device (as would human analysis do) represents a limitation to generalization.

As a consequence the document analysis research community (as well as the broader image processing community) has quite well identified this problem as related to the adaptability of the interpretation device. To try and address it, knowledge based alternatives have been developed in the hope of achieving more versatile analysis processes.

An illustration if this transition is the work by [Devaux et al. 1998] which consists in transforming 2D ANSI representation of 3D objects (containing dimensioning lines, orthographic projections, etc.) into full 3D representations. Although their work can still be considered as very similar to the bottom-up approaches, it clearly distinguishes itself from them by representing analysis knowledge as rules ${ }^{3}$. An illustration from their work is shown in Figure 7.

\footnotetext{
${ }^{3}$ We are only considering [Devaux et al. 1998] from the perspective of graphical document analysis, and the ways in which it extracts higher level information from image pixel data. It does not intend to be a state-of-the-art reference to the problem of reconstructing 3D shapes from 2D projections. This problem has been addressed elsewhere and goes far beyond image analysis; it does not fall within the scope of this handbook.
} 


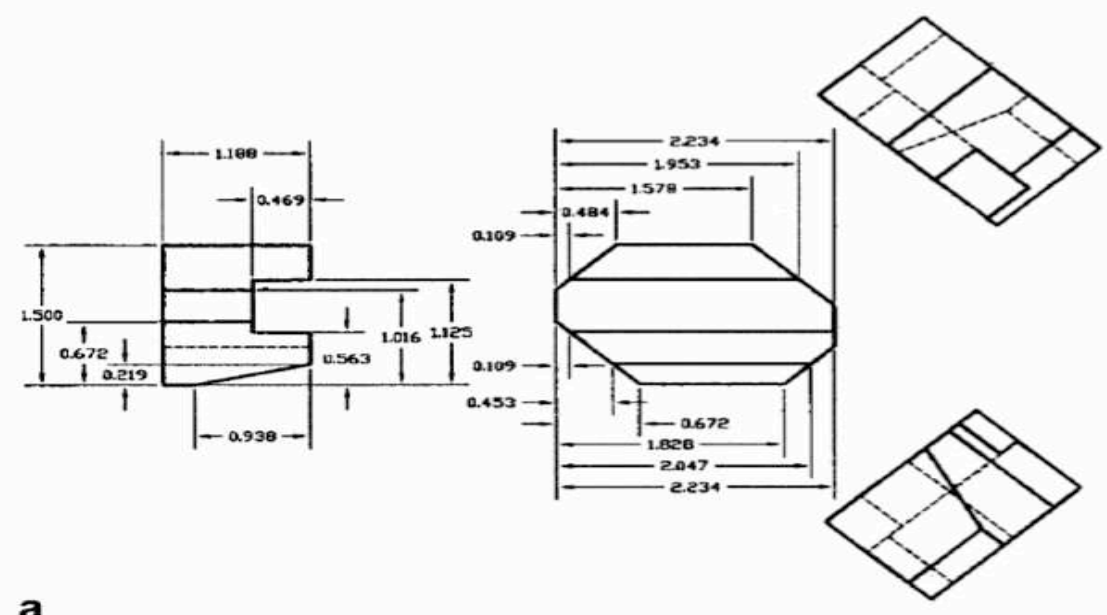

a

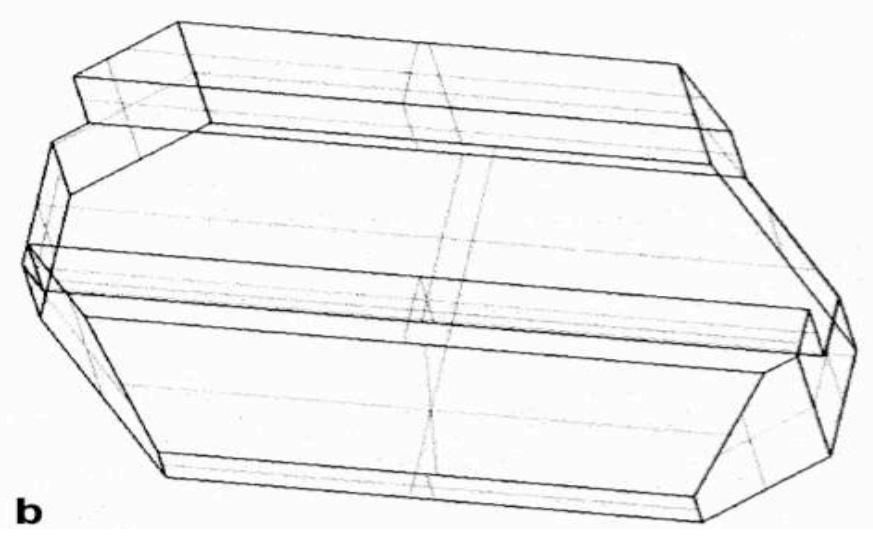

Figure 7 from [Devaux et al. 1999] obtaining a full 3D representation from a set of 2D ANSI orthographic projections.

\subsection{Knowledge based approaches}

In order to solve the difficulties mentioned in the previous section, mainly that low level segmentation and extraction methods can be used off-the-shelf, but that domain knowledge and analysis "intelligence" is embedded in the underlying algorithms, many references in the literature consider knowledge-based approaches. This kind of approaches generally tries to solve the adaptability and genericity problems by formally representing some of the contextual knowledge needed for the interpretation. It thus becomes "externalized" from the analysis process, where it was implicitly embedded, before. This externalization of knowledge the together with dynamic links between the process and the knowledge database opens the possibility to implement flexible and adaptable interpretation devices implementing a generic analysis that is capable of adapting itself to contextual information. This section provides an overview of some of the best known knowledge models used in graphical document analysis.

\section{About knowledge modeling}

Some authors propose using models of different knowledge categories that would contribute to the analysis process [Adam 2000] and that these models be formalized as much as possible as to obtain a truly adaptable and context independent interpretation system. A classification of the required knowledge in 4 categories can be found in [Ogier 2001]:

- The most obvious category concerns descriptive knowledge. It covers the knowledge over the physical or conceptual domain (semantics) represented in the document on the one hand, as well as the graphical conventions (semiotics) and the rules that govern them (syntax) to represent concepts. It may also include semantics of the document's conventions like captions, legends and references. Most often, however, it represents the rules for representing objects within the document and generally relies on 
structural/syntactic representations, such as graph, trees, grammars, semantic networks or ontologies, some of which have already been described in Chapters 5.1 and 5.2. The representation of this knowledge allows to describe the hierarchical organization of elementary primitives, as well as their topological and geometric relationships. This hierarchical description then allows to further define semantic consistency rules that can be used to check whether the information generated from a bottom-up strategy is consistent or not.

Furthermore, it can also be used to define the sequences of tasks and sub-tasks to be run in order to progressively extract the information from the image and organize it according to the model. Recent developments [Raveaux 2010] have started introducing ontologies to formalize this knowledge, since it allows not only to model hierarchies of concepts, but also the relations that connect them.

- Another category covers the Image Processing operator's knowledge. It corresponds to the knowledge that is used by an expert to construct the analysis process in the context of image processing or pattern recognition problems. It is related to the behavior of the various image processing operators that are used to implement the analysis strategy: they allow to describe in which context these operators are most appropriate, how they must be tuned versus a specific context,... For instance, they can correspond to the choice of the best image processing operator for segmenting a texture, or to the best couple (features vectors/classification process) that has to be used for recognizing a specific symbol. This knowledge is generally implicit and is rarely formally modeled. It finds itself embedded into the way algorithms are combined together ( $c f$. next knowledge category) to form the overall analysis process, what parameter intervals are used and how they are obtained, which error or decision thresholds are used etc. However many papers mention the necessity to integrate this aspect when trying to implement generic systems. One of the steps in the direction of capturing the operator's knowledge, although not sufficient by itself, is to provide clear descriptions of input and output parameters and execution semantics of algorithms [Lamiroy \& Lopresti 2010]. A more formal experiment toward integrating expert operator knowledge can be found in [Clouard et al. 1999] although, strictly speaking, it does not fall within Graphics analysis, it does, in a general sense, apply to the issues described here. It presents the BORG system, aiming to generate image processing programs and for which the proof of concept was established for cytology in medical imaging. Besides the grammar-based control mechanisms developed in the next sections (ANON, ADIC, etc.) and implementing selection strategies for finding the correct rule set to apply, BORG also allows to integrate quality measures to the image analysis steps expressing conditions like "if the standard binarization algorithm gives rise to too many small connected components, revise the set of used thresholds in a previous step, or switch to and alternate binarization algorithm". The approach is not an image analysis method in a strict sense but a image analysis generator (i.e. given constraints, knowledge and a set of training images, it will generate an image analysis program satisfying the given conditions and operating on the provided class of images)

- Strategic knowledge a complementary level of implicit knowledge that is used by the image/pattern recognition expert when implementing an interpretation strategy. It deals with with the sequential ordering of a set of image processing operators in order to reach the analysis goal: how to sequence a set of image processing operators and pattern recognition processes, in a particular context and in order to achieve a specific level of interpretation. This kind of knowledge far more difficult to formalize and is of a much higher level than the previous one, since it concerns the way to organize the process, and not exactly how to tune each of them. The formalism that can be used for modeling this kind of knowledge can be inspired from Petri networks or serious games. 
All these knowledge categories are implicitly involved in the building of an analysis device and we consider that the genericity of analysis systems necessarily requires some level of formalization, and external representation falling into these categories. While there is currently no existing consensus or formal theory on how to ideally achieve this, there are however tentatives in this direction and the mentioned levels of knowledge are more or less formalized in hybrid systems, which are presented below.

\subsection{Hybrid approaches for graphics analysis}

Hybrid approaches use a subset of the knowledge categories mentioned in the previous section for analyzing technical documents by leading the low level processes as a function of the context. From a historic point of view, two approaches constitute interesting contributions to these approaches and can be taken as representative tokens of a more comprehensive state-of-the-art.

\subsubsection{ANON and Grammar Based Derivatives}

One of them was proposed by Joseph [Joseph 1992] and concerns mechanical engineering drawing analysis. It was called ANON and used the "cycle of perception" proposed by Neisser, the basis of the approach being a continuous loop in which a constantly changing world model direct perceptual exploration, determines how its finding are to be interpreted and is modified as a result. In ANON, this role is taken by an instance of one of a number of schema classes. The system is structured in three layers in order to separate spatial and symbolic processing. The first is composed of a large image analysis library associated to both search-tracking functions and a management processes. The information extraction is adapted to the context by the second level, the "schema" (prototypical drawing construct), which receives the entities from the lower layer and interprets the result as a function of the current schema. A cycle of hypothesis verification is thus proposed by the schema to the control system (highest layer). On each cycle, the controlling instance invokes appropriate members of ANON's library of image analysis routines and informs a higher level control of the results of its actions. This control system analyzes the proposition as a function of the current state of the proposed schema and eventually modifies it. Applied in the context of graphic recognition, the system presently maintains classes corresponding to solids, dashed and chained lines, solid and dashed curves, cross hatching, physical outlines, junctions, letters, words, witness and leader lines and certain restricted forms of dimensioning. Each schema instance represents a particular example of some prototypical drawing construct.

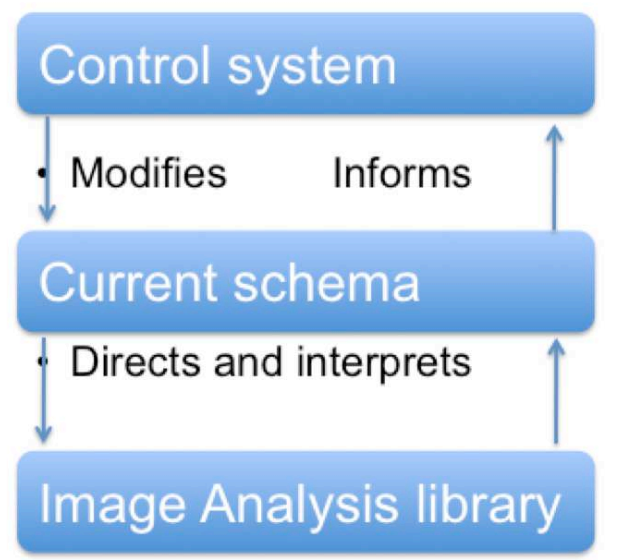

Figure 8 Control structure for ANON, as represented in [Joseph 1992]

ANON's control module comprises a set of strategy rules written in the form of a grammar. These rules define methods by which high level drawing entities may be obtained by hierarchical combination of low level constructs. On each cycle, the control system determines an appropriate 
modification to the current schema. Modifications may correspond to an updating of an internal variable, the adding of new sub-parts, or the replacing of the instance with a new one representing a different type of construct. Strategy rules, like string grammars, describe acceptable sequences of events.

The results obtained in [Joseph 1992] are shown in Figure 9, and clearly illustrate the limitations of the knowledge-based approaches in their beginnings. They very difficulty account for noise or configurations slightly deviating from the conventional representations.

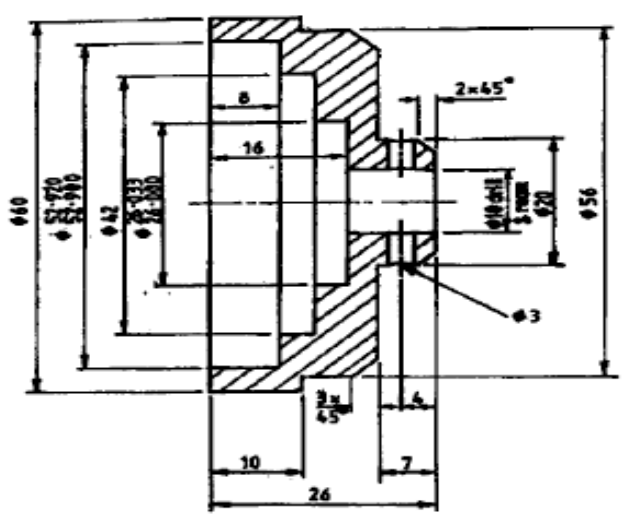

(a)

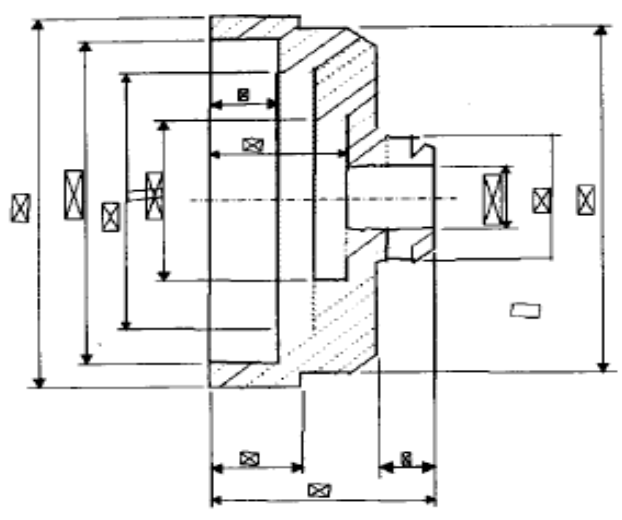

(b)

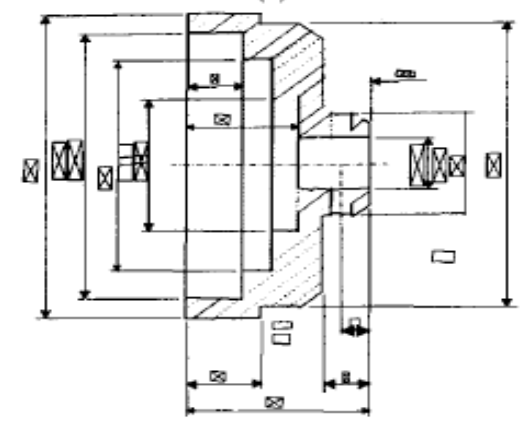

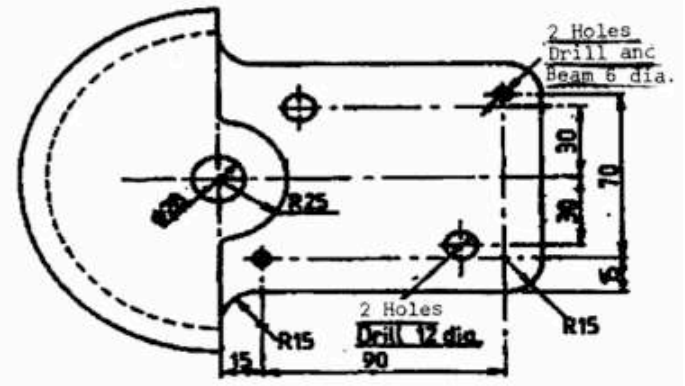

(a)

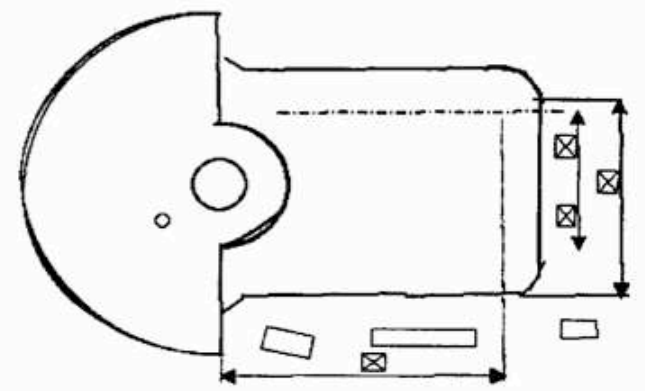

(b)

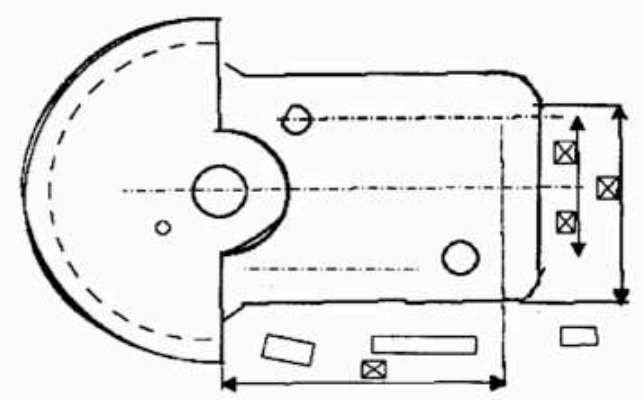

Figure 9 as reported in [Joseph 1992] the original images are on top (a), and the raw output is given on line (b). Line (c ) shows manually corrected results. Note the difficulties in interpreting hashed areas and connect broken or intersecting lines.

The knowledge directed image analysis and the construction cycle according to the context are two interesting concepts which are applied on 15 different schema classes.

A similar approach is ADIK [Pasternak 1994]. Its approach is very much related to syntactic symbol recognition and addresses the interpretation of technical diagrams by representing visual knowledge in the form of a grammar, expressing the various relationships and hierarchies between primitives and shapes as well as tolerances on allowable perturbations. The approach is conceptually similar to ANON, but is more flexible where its grammatical expression is concerned. 
The LR-grammar is extended with "placeholders", "triggers" and "constraints" which give it more possibilities to express local contextual conditions, making its behavior on triggering interpretations more flexible. Figure 10 gives some examples of detection results, and the resulting exploration tree that results from the interpretation of the drawing.
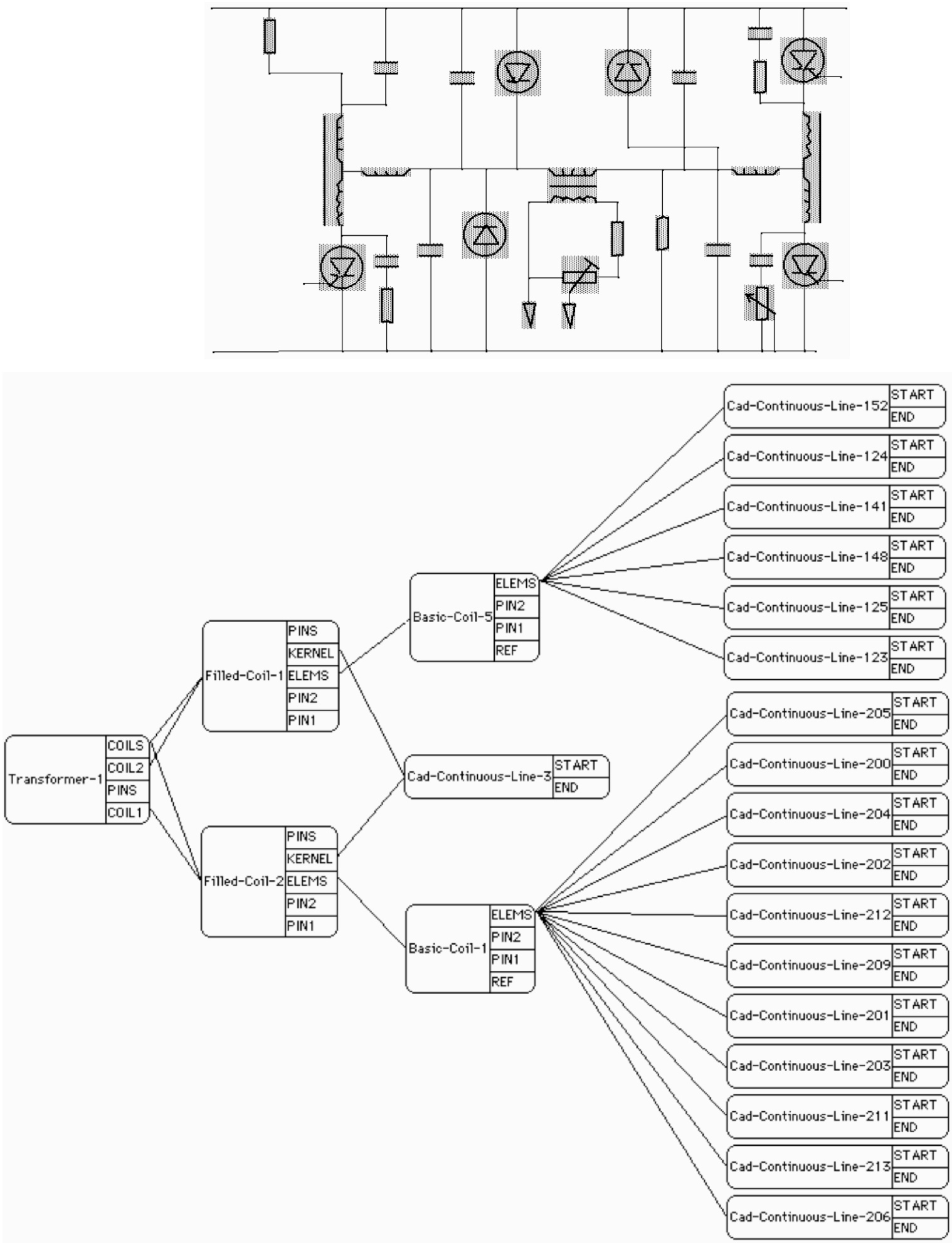

Figure 10 taken from [Pasternak 1994] showing its results on electrical wiring diagram analysis and the resulting interpretation tree that results from the interpretation process, connecting high level concepts (transformers, for instance) to image features (continuous lines).

In the same category, den Hartog [den Hartog 1996] proposed a mixed approach based on a topdown control mechanism associated with bottom-up object recognition. The system decomposes the 
binary image into primitives (and not vectors) having a good morphological representation of the information and uses template matching to recognize each of them. Then, contextual reasoning is performed based on a loop that includes inconsistency detection and search action generation in a region of interest (ROI). The control system defines an ordered search action list to search for a specific object type in the ROI. The user specifies priorities to define the most important search actions and to assign priority to the relationship between objects. A consistency test is applied to each recognized object in order to verify the hypothesis defined at the system's top level as a function of knowledge of the object to recognize. The knowledge framework of the methodology relies essentially on spatial relationships between primitives, without integrating and describing hierarchical relationships. In the case of particularly complex documents, this kind of system is penalized because of the drastically increasing number of relationships and the necessity to generate new search actions for the "designed objects".

More recent work, revisiting the previous approaches, can be found in [Lu et al. 2009]. They identify several shortcomings, the main ones being related to the fact that only graphical constraints are explicitly modeled, while in reality, technical drawings are also governed by implicit composition rules. This has lead to over-investigate approaches that are essentially "linear" in their approach to combine graphical information and achieve interpretation from a set of pixels and for which non-shape domain information is not explicitly represented, or at best, according to the authors, embedded in complex rule sets.

$\mathrm{Lu}$ et al. [Lu et al. 2009] therefore suggest using the explicit geometric shape definitions as entries for which implicit (in the sense that they are implicit for the human interpreter; meaning they need to be made explicit for an automated process) composition rules and representation conventions are used for guiding the analysis process and to check consistency or remove ambiguity. Their architecture is based on a knowledge interpreter, a knowledge parser, and an entity searcher; very much like ANON [Joseph 1992]. Using the assumption that automatic interpretation is composed of a series of condition driven processes, these conditions are represented as knowledge descriptors either addressing representation issues (recognition) or interpretation issues (control).

They identify four levels of interpretation targets: project, drawing, engineering entity and graphical primitive for which knowledge is represented in EBNF (Extended Backus Naur Form), all of which have external (purely graphical representations) and internal states (based on contextual and composition rules).

As shown in Figure 11, the method is capable of detecting "similar" items, not only based on shape, but also based on actual semantics. 


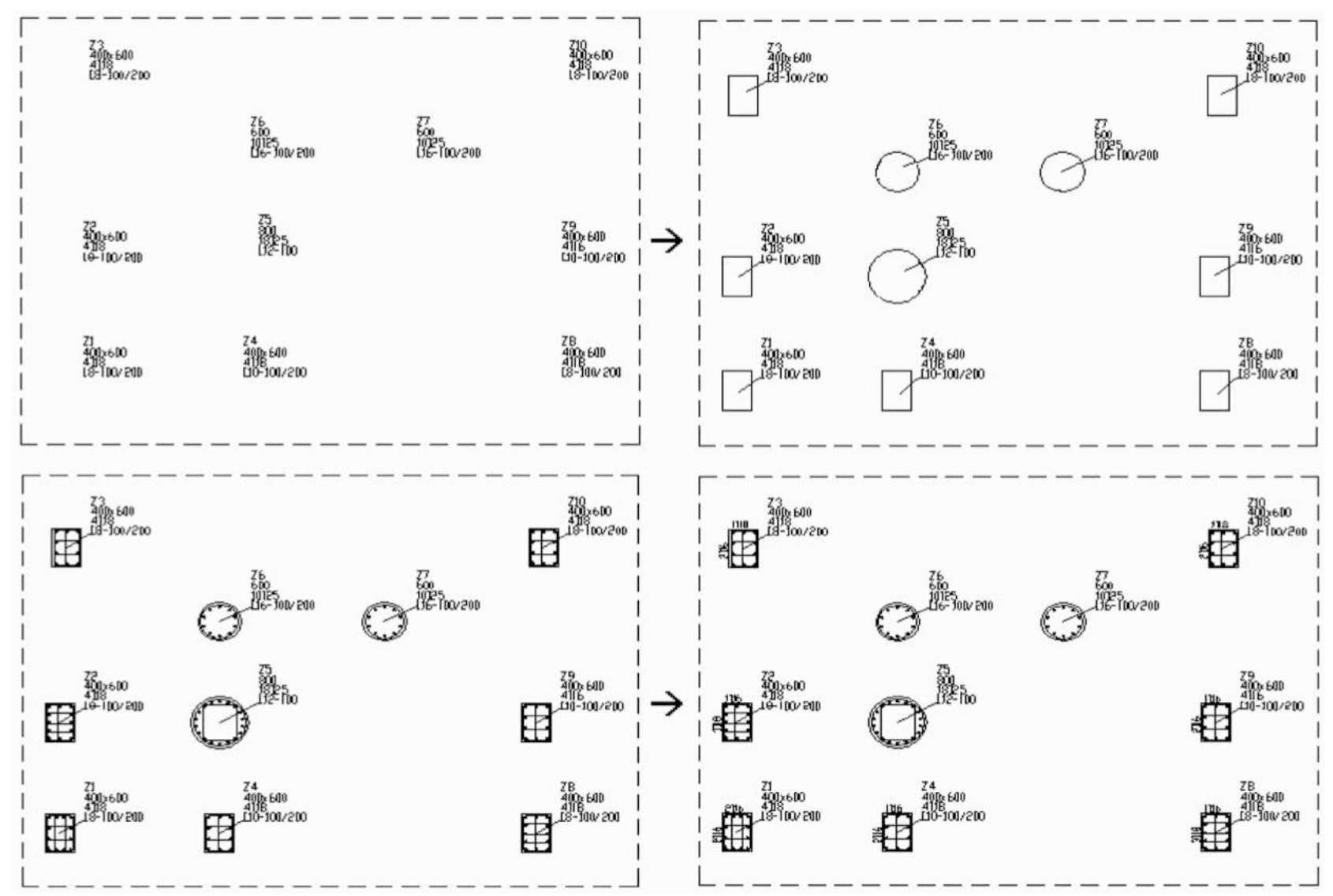

Figure 11 taken from [ $\mathrm{Lu}$ et al. 2009] looking for reference source columns in technical construction blueprints; starting with an initial example, and progressively expanding its knowledge tree to find all occurrences in the drawing.

The approach remains very sensitive to exhaustive visual modeling and errors introduced by noise on the one hand, as well as incoherency or missing information (local non respect of conventions).

Among other relevant work, it is important to mention Coüasnon's DMOS system (Description and Modification of Segmentation) for analyzing structured documents, and which can also be applied to graphic documents. The aim of this system is to design a generic recognition system, being able of producing either general or specific systems. DMOS system is made of a grammatical language (Enhanced Position Formalism-EPF) and an associated parser able to deal with noise. As for the previously presented systems, the main principle of DMOS is to separate domain knowledge from source program, in order to develop the adaptivity of the system. Actually, DMOS relies on a compilation phase, which, in its turn, builds an adapted recognition system, on the basis of an EPF description of the expected document structure.

As the authors state in [Coüasnon 2006], "This method has been successfully used to produce recognition systems on musical scores, mathematical formulae and even tennis courts in videos. This. Therefore, for a same kind of document like table structures, it is possible to define with EPF, more or less specific descriptions to produce more or less specific recognition systems. For example, we have been able to produce a general recognition system of table structures. It can recognize the hierarchical organization of a table made with rulings, whatever the number/size of column/rows and the deep of the hierarchy contents in it, as soon as the document has a not too bad quality (no missing rulings for example). We will present the way the description is done using EPF to be general enough to recognize very different table organizations. With the same DMOS generic method, we have also been able to easily define a specific recognition system of the table structure of quite damaged military forms of the 19th century. This specific description was necessary to compensate some missing information concerning the table structure of those military forms, due to 
a very bad quality or hidden part of the table. This system has been successfully validated on 88,745 images, showing that this DMOS generic method can be used at an industrial level."

\subsubsection{Context Modeling and Ontologies}

Another hybrid approach is the system described in [Ogier 1998] for map interpretation. In this system, features are grouped together to constitute primitive objects then these objects are assembled together to compose a larger object in the hierarchy and the process continues until it reaches the most global object which is the map itself (Figure 12)

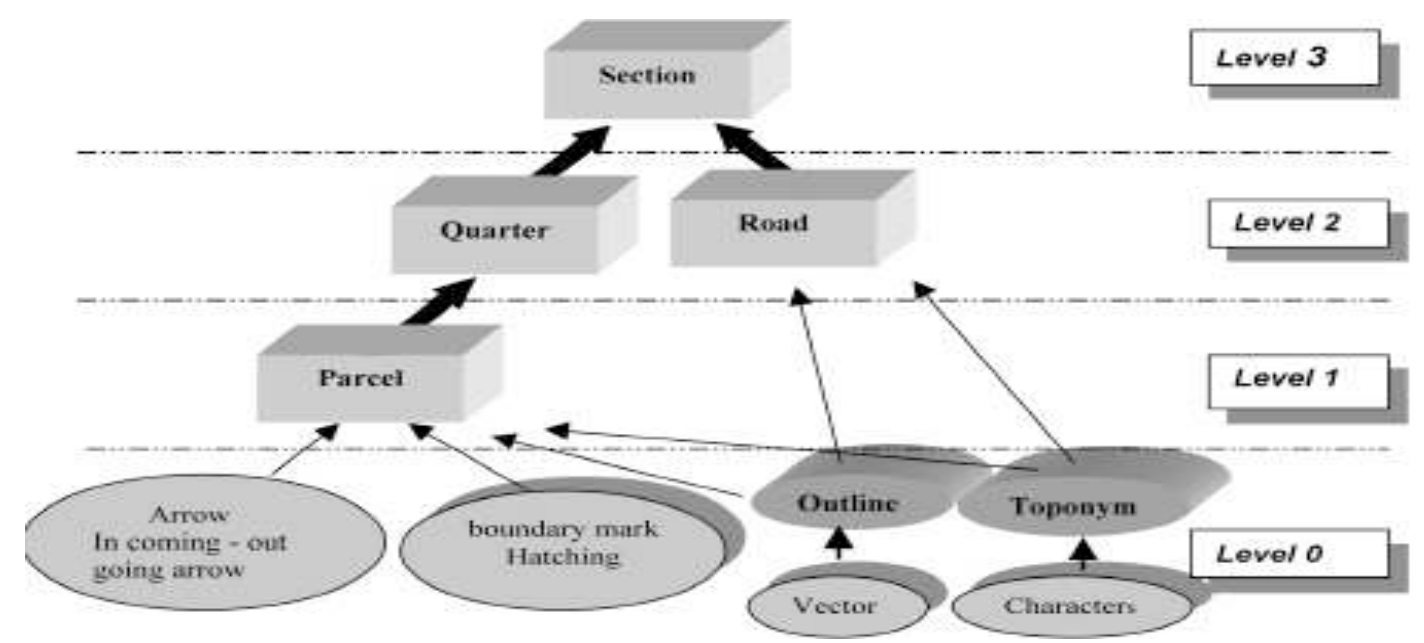

Figure 12 : taken from [Ogier 2000-b] illustrating a model of knowledge representation in the context of French cadastral maps

Although this formulation is not fundamentally different than the ones expressed previously (bottom-up and/or parser-based), the focus lies more on the fact that consistency checking is performed at every level in the hierarchy. Recognized objects are analyzed to verify if they are internally and externally consistent with each other. For example, a parcel is composed of segments to set up the outline, it has a number or an arrow, and it can involve a hatched area and symbols. Internal consistency means all the components composing the object are successfully detected, if not, a forward heuristic rule is used to correct this situation by re-extracting features in this region after modifying and relaxing the parameters of the low-level image processing tools. On the contrary, external consistency takes into account the neighborhood of the treated object. If an object has all the components and responds to the semantic of the considered level, it is defined as an internal consistent; furthermore, if all the objects adjoining it are all internally consistent, this object will equally become more reliable through the construction of the superior hierarchical level (the parcel by the block, for example). It is then called externally consistent. This approach is summarized in Figure 13. 


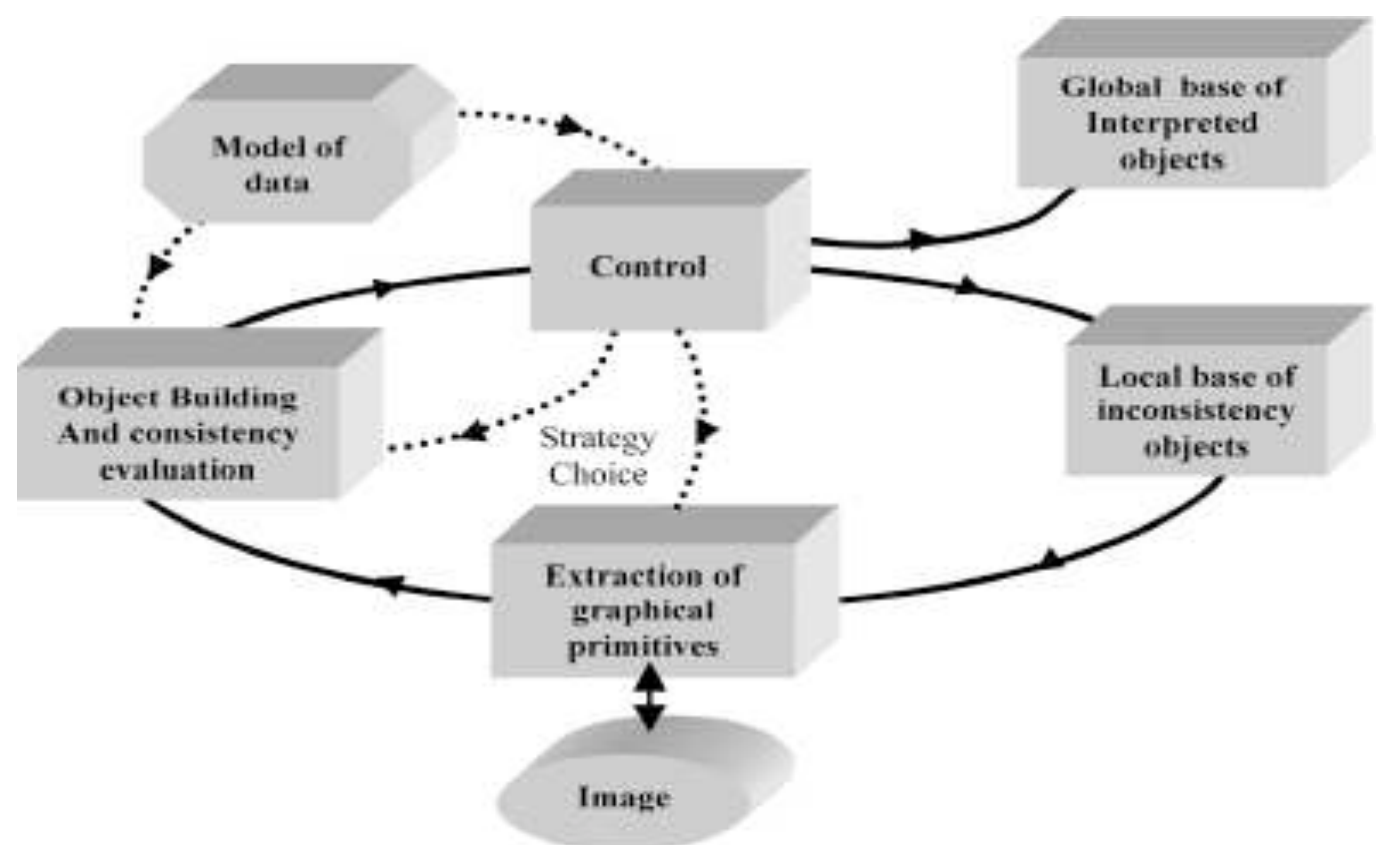

Figure 13 : taken from [Ogier 2000-b] illustrating a cyclic strategy for the interpretation of French cadastral maps

One of the more recent approaches was proposed in [Raveaux 2010]. In this thesis an object extraction method from ancient color maps is proposed. It consists of the localization of the frame, text, quarters and parcels inside a given cadastral map. First, a model of what visually characterizes a cadastral map is defined by combining knowledge from various domain experts: historians and architects on the one hand, image analysis professionals on the other hand. Next, dedicated image processing tools aim at locating the various kinds of objects laid out in the raster image. These especially designed detectors can retrieve different components such as characters, streets, frame, quarters and parcels. Thereafter, this information feeds a higher level which elaborates a graph structure where, nodes refer to the presence of objects found during the detection step and edges represent the spatial relations between them. Terms, words and appellations to qualify node and edge labels are so called concepts. All concepts have been previously modeled by the domain experts, and are represented in an ontology containing the vocabulary and the description logics of each element required to model a cadastral map. Therefore, the produced graph can be seen as a particular instance of the generic map model. On the other hand, given the relatively "bottom-up" extraction method used to obtain the graph, the latter is not constrained by the ontology and variations which are non-conforming to the knowledge base may have been introduced into the graph structure (due to defects in the detection algorithms, noise in the images, unexpected shape variations, etc.). As a consequence, a higher level of representation is required to to determine to which level the extracted graph conforms to the expert knowledge. The structure of the graph is analyzed with the joint use of a cadastral map ontology to re-engineer a meta-model corresponding to the instance data. In a last phase, the meta-model corresponding to the instance data is compared with the meta-model defined by the experts. This comparison is carried out thanks to a graph matching algorithm. An overview of the main actions carried out at this stage is shown in Figure 14. 


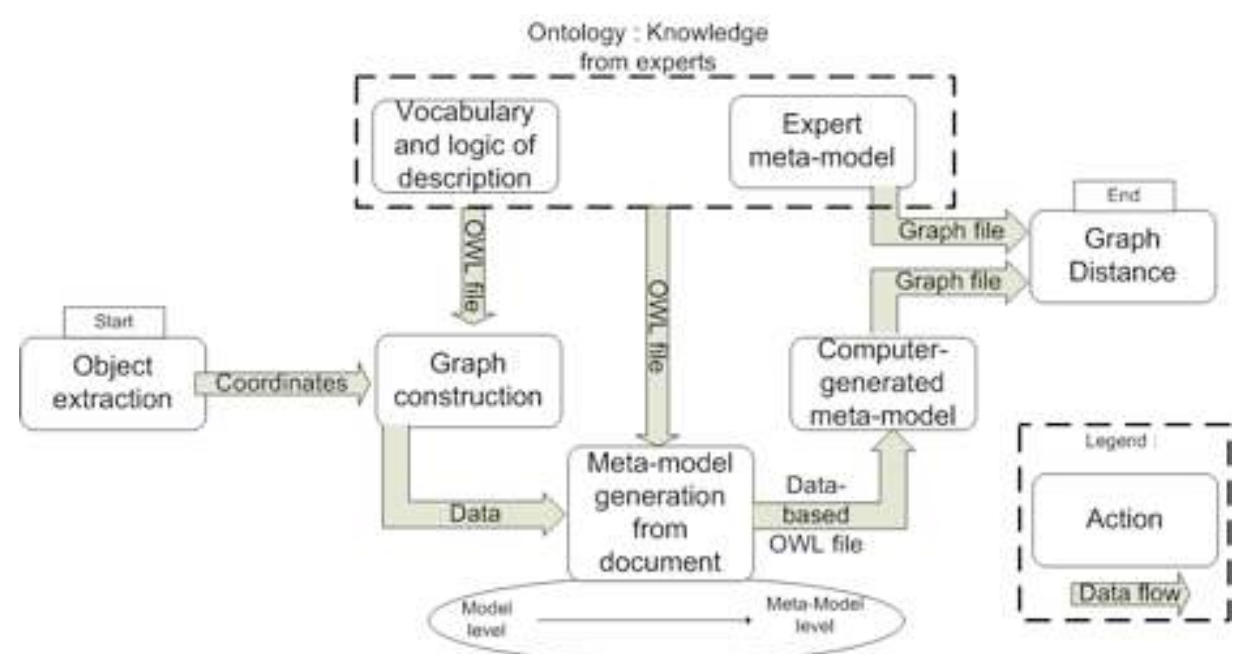

Figure 14 Data flow process for meta-model inference from a model, taken from [Raveaux 2010]

\subsection{Discussion and Limitations}

\subsubsection{General Discussion}

Although the knowledge based approaches described in the previous section seem intellectually much more satisfactory and more enhanced than classical approaches since they try to dynamically link knowledge, image analysis techniques, pattern recognition tools, and document interpretation contexts, very few production-ready systems or significant technological breakthroughs have been reported since 2000. [Kasturi 1996] contains a good state-of-the-art review of the main available techniques and tools for the analysis of technical and cartographic documents and subsequent publications have not really contributed to fundamentally change the state-of-the-art.

Exception can be made for the recent evolutions involving the development of ontologies and web semantic approaches. They offer some interesting alternatives that allow to better formalize knowledge and render them quite versatile for use in analysis systems. However, considering the high level of complexity of these problems, even if scientific communities agree on the importance of the development of generic interpretation systems, and the necessity to dynamically connect knowledge management and analysis scenario, one must admit that there is still no generic system allowing to solve any interpretation problem. One of the reasons may be that ontologies themselves (or any other formal knowledge representation) are only partially capturing the underlying complexity of the required knowledge by requiring that it be expressed within the boundaries of computational description logics, thus shifting the intrinsic difficulties just a level further without actually addressing them in full.

Although there is no formal evidence of the following assumption, the graphical document analysis advances seem to have reached a plateau where end-to-end generic interpretation systems are concerned. The collection of concepts and methods coming from compartmentalized research communities that are required to be integrated with one another to to produce full document analysis seem to resist to all efforts trying to remove human ingenuity. Therefore, current tendencies show the evolution towards strategies which are easier to implement, based on user interaction, or based on partial interpretation strategies. Among these partial interpretation strategies, many of them rely on spotting based concepts, that consist in offering navigation services into document database, without systematically interpreting the document content. These research axes appear to be very promising since they represent a good trade-off between efficiency, genericity, and automation. Some of these approaches integrate user interactions for the management of knowledge in order to dynamically adapt to new interpretation contexts, without the need of having them formalized. A short overview of these systems is given in the following part.

\subsubsection{Cookbook \& Practical Tips for Graphics Interpretation}


The essential remaining question that we need to address in this chapter is "What do I do with my particular interpretation problem?" for the user who wants to implement some of the tools reviewed in this handbook.

We have already stressed on multiple occasions throughout this chapter: there is no standard answer to the question. There are, however, some decent rules of thumb, that may guide the interested reader to a practical, operational and efficient compromise. The following table gives an overview of configurations that are likely to occur, based on how much one knows about the graphical data at hand on the one side, and the intended interpretation context on the other side.

Control over Interpretation Context (horizontal)

$v s$.

Control over Data Variability

(vertical)

Low

Data may contain relevant patterns but they cannot be determined in advance and data of interest may be hidden in stream of potentially irrelevant documents.

Medium
Data can be
assumed to obey
general visual
rules, but
variations in
shape or noise can
be important and
are difficult to
model. The
application
context is such
that outliers
containing
completely
irrelevant data are
rare.
rare.

\section{High}

Data falls into a very well understood and formally modeled scope, noise and
Low

Interpretation context cannot be formally determined: general public and nonspecialist users.

No reports exist of

proven valid approaches in this very open context. Current consensus is that the most appropriate way of dealing with these classes of problems is to use weakly supervised classification and user relevance feedback to implement a symbol spotting approach ... and hope for the best [Rendek 2006]
This situation corresponds to particular cases for which similarity between data can be expressed through statistical descriptors as well as visual key point approaches in the context of information spotting. This kind of approach has been recently used in the context of historical document indexing [Thi 2011] in the case of weakly structured graphic images. User interaction should be used to characterize Highly controlled graphic data most often comes from very well controlled production contexts and obeying to a very

\author{
Medium \\ Interpretation context \\ can be formalized in \\ some way and/or public \\ or users are assumed to \\ be knowledgeable to \\ some level.
}

This is one of the harder cases, where there is a fairly well, although insufficiently formalized

interpretation context, but without sufficient visual support in the data (i.e. there is a large semantic gap) In this case, the most appropriate approach is likely to investigate further whether there are classes of interpretation contexts that can be better specified and reduce the problem to a more constrained one.

As far as we know, this context is very rare and there is no real reference for such a context.

Since the graphic data can be fully modeled and noise and deformation levels are known the appropriate image description tools
If the interpretation context can be formalized in explicitly it is possible to establish a set of constraints the data must fulfill (although they need not necessarily be expressed in visually verifiable ways). This can in its turn guide top-down analysis strategies, on the one hand. On the other hand, the presence of specialized users can also lead to a two-stage coarse-to-fine approach where supervised learning techniques can be trained to reduce the number of irrelevant documents first, and then separate the data "manifold" into "submanifolds" of lower variability.[Ogier 1998] In this situation the focus of the analysis process will be on establishing how the formal interpretation knowledge projects onto the approximate and noisy (yet globally coherent) graphical data. This is probably the most active area in the current state-of-the art.[Coüasnon 2006, Raveaux 2010] are excellent examples.

In this configuration the level of available formalization of both the interpretation and the data does not require a complex level of knowledge representation. A custom-
Invariants for given variability on data

For this category of graphical data the best approach is to use statistical learning over an as large set of possible relevant descriptors.

There is no guarantee that the combination of classifiers and descriptors will eventually capture all possible instances of relevant data.

Statistical evaluations of the power of discrimination of descriptors should be used [Rosenberger 1998]

When data is only approximately formalized yet most of it is relevant, the current state-of-the-art consensus is to use nonor semi-supervised classification techniques to determine optimal descriptions of the graphical data, e.g. by using visual bag-ofwords. Depending on how much is known about the interpretation context, the supervision of the classification techniques and/or their $a$ posteriori validation can be more or less elaborate and efficient.

Given the high accuracy of knowledge about how the data behaves, robust and reliable visual descriptors can be easily extracted from the 
deformation levels are within well established bounds, outliers are rare.
Invariants for given class of interpretation contexts. well established visual language. It seems contradictory to combine this with a undetermined interpretation context, but there are situations where this makes sense. For instance, electric wiring diagrams or architectural floor plans may be used for a very broad variety of interpretation contexts. The most reasonable approach here is to consider "retroconversion", without trying to capture the end-user's interpretation intent, but by trying to express the visual information into a higher level formal description that can then be used as a support for a userdriven analysis process. [Kasturi 1995]

In this context it is impossible to make any assumption about what final interpretation is required, and needs to be left to subsequent treatment or analysis; either this is done by the user itself, either enough is known about the visual description language so that a formal description of the document can be extracted for further automated analysis. can be used to fully characterize the data. According to the available interpretation knowledge the user can be presented with

classes of visual information or partial interpretations with respect to the level of available interpretation contexts' formalization. Semi-supervised learning or user relevance feedback can be applied to converge toward a useracceptable interpretation. Fully automatic interpretation remains out of reach. [Lu et al. 2009] tuned (probably bottom-up) approach and hard-wired approach is very likely to be the most efficient and costeffective. [Dosch 2000]
The existence of a very well known and formalized interpretation context, as well as specialized users, offers the possibility to fully express acceptation/rejection criteria of interpretations. Depending on what quality of data it is applied to these acceptation/rejection criteria can more or less be related to visual information. document images.

Depending on what kind of interpretation knowledge is available, this visual (low-level) interpretations can then be more or less related to higher level interpretations, either fully automatically or with some user interaction, when possible.

\section{Evolutions and Challenges in Graphics Interpretation}

\subsection{Recent evolutions: learning, spotting and indexing for navigation into graphic document repositories}

Because of both the theoretical and practical considerations mentioned in the previous section related to the difficulty of developing generic analysis systems that would be able to manage any kind of document within a broad range of representations, recent approaches, principally developed during the last decade, try to approach analysis from another viewpoint. Rather than to aim for a fully automated analysis process and subsequent interpretation, with the difficulties of capturing all contextual knowledge, the research focus has shifted to leaving part of the analysis to a human interpreter, and to offering efficient tools for handling large volumes of documents, mainly using "intelligent" indexing strategies. 
One of the main turning points introducing this paradigm shift can be attributed to [Tombre 2003] spurring investigations towards the idea of "spotting" or [Nagy 2004] insisting on human interaction. Their overall observation is that rather than trying to fully represent contextual knowledge for solving interpretation problems, three main substitution strategies may prove just as effective:

1. example-based or supervised learning and classification techniques can be used to replace interpretation context modeling (although there are pitfalls to be avoided if the sample population is inadequately chosen [Nagy 2004]);

2. spotting and indexing can be used to (partially) replace full contextual interpretation by guiding a human interpreter rapidly to documents or document parts that have a high probability of fitting her interpretation;

3. human interaction should be much more seen as a continuous part of the analysis process, rather than just participating in the knowledge modeling phase (e.g. by dynamically influencing classifier decision boundaries, or indexing feature selection through relevance feedback)

As an illustration, we are developing the example of an analysis system using spotting techniques and indexing. The idea of spotting essentially consists in favoring recall over precision by proposing realistic navigation and retrieving services on large document corpora, fine-tuned on the requirements of a specific use case (usually given by the organizations managing the corpora), without systematically running costly full recognition processes (i.e. both high precision and high recall). One of is main advantages is that avoids trying to interpret the whole document (which, for specific applications is not really useful, anyway) and allows a possible second stage process to focus on the interpretation on relevant spotted objects. Therefore, this kind of approach allows to simplify the analysis process, since it can use alternative detection and recognition processes which rely on less complex data representations and pattern recognition strategies. Furthermore, from the knowledge management point of view, this kind of system does not necessarily require exhaustive formalization them since most of them can be dynamically caught through man machine interactions, or relevance feedback. The global schema of such a spotting system is presented in the Figure 15.

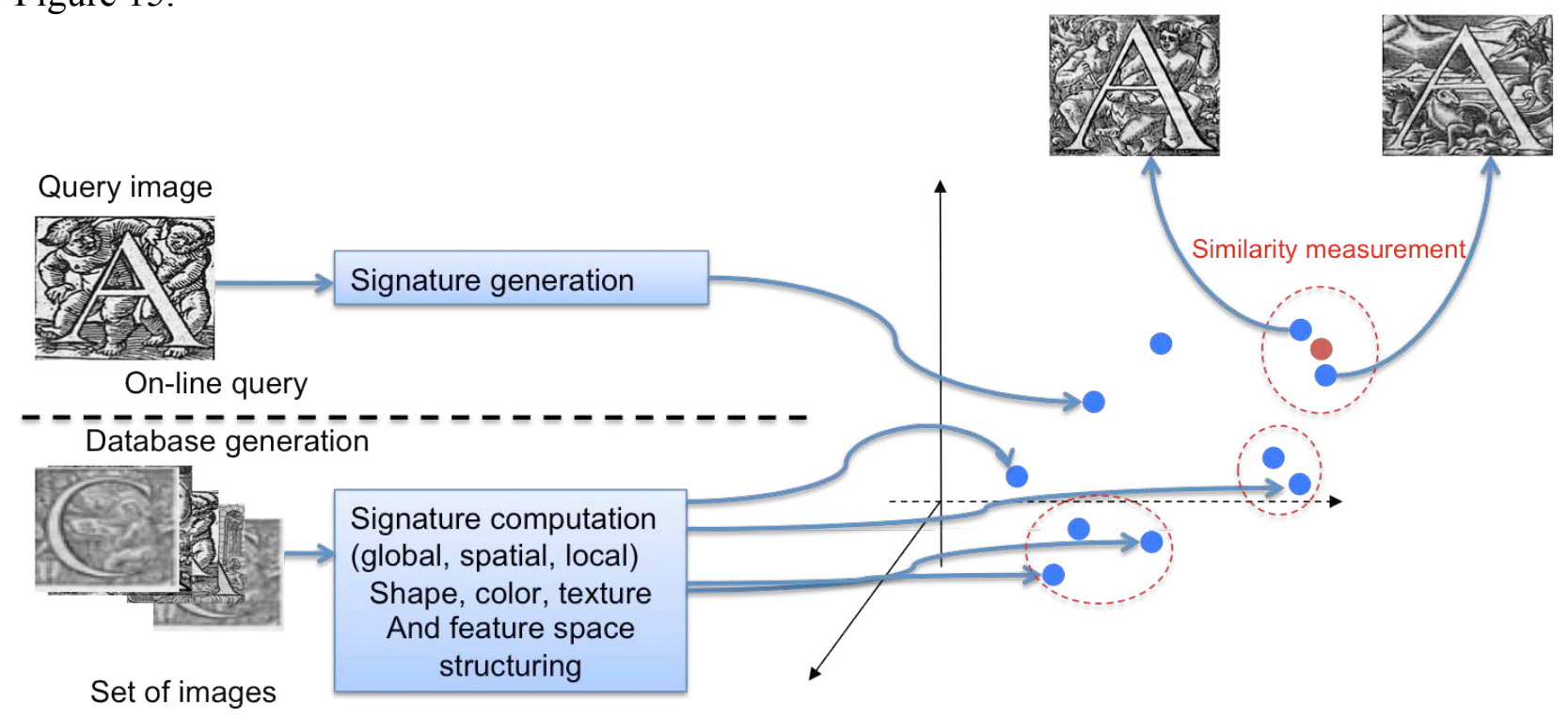

Representation space

Figure 15 Principle of signature computation and information spotting in the context of drop caps spotting This kind of system is generally composed of two main parts, one which is off line, and another which is on-line.

The off line part aims at analyzing the content of documents by extracting some features allowing to characterize each of them in a unique way. Until the beginning of 2000 , the features that were 
used to describe documents generally relied on classical pattern recognition based descriptors, i.e. based on statistical of structural approaches described in previous sections of this handbook. More recently, bag of words approaches were proposed, trying to characterize document contents without necessarily describing them on the basis of human knowledge.

The on-line part aims to proposing interactive interfaces allowing the user to retrieve documents on the basis of queries, which can either be expressed on the basis of keywords or on the basis of images.

\subsection{Challenges in Graphics Interpretation}

The main conclusion we can draw from the previous sections is that there is no actual commonly agreed set of best practices or globally adopted methodology for complete analysis and interpretation systems. As a matter of fact, the research community has gradually abandoned the investigation of end-to-end applications in this domain, focusing more on sub-parts like recognition and indexing, segmentation or spotting. This is the main reason why many of the references in the previous sections are relatively old. While the results of these described approaches are quite interesting in their respective application contexts, it becomes less easy to really assess their value in from a more general viewpoint: how do they adapt to other contexts, how do they perform with regard to recent developments in lower level treatments, etc.? The result is that most interpretation and analysis methods remain very context specific, and that, therefore, analysis problems are handled on an ad hoc basis. Therefore, the main challenge for the graphic interpretation community is to establish a classification of its methods and low-level approaches described in the previous sections, and to relate their appropriateness to higher-level interpretation contexts. As made clear through the overview given in this chapter, and based on the results presented in the previous chapters, there is a significant gap between the performance of individual graphic image treatment and recognition approaches and the performance of full analysis methods. In this chapter the focus has been set on knowledge modeling as a means to bridge this gap, and several partially successful approaches have been developed in this domain, over the years. However, effective conclusions still need to be drawn from these experiments and there is no established consensus on good practices or better choices in specific application contexts.

The main challenges for graphics analysis therefore are related to performance analysis on the one hand, and context characterization on the other hand, as well as fully integrating the human user in the analysis and interpretation process, helping to focus the knowledge modeling or information characterization through relevance feedback, for instance.

It remains an open questions whether this is actually a realistic goal. Since there is no recent published work in what interpretation exactly means or entails from a Machine Perception point of view, the problem of measuring the state-of-the art remains open. However, when broadening the scope beyond Machine perception, into formal semantics and model-checking on the one hand, and reaching out even further into linguistics, and even metaphysics, it does not seem absurd to try and relate recent advances in those domains concerning semantics and interpretation to what has been described in this chapter. While automated interpretation of perception data is a very ill posed problem in the current state-of-the art, trying to formulate it in a more abstract way will probably show a number of limitations related to tractability and decidability and therefore allow the graphical document analysis domain to better grasp the reasons behind the currently observed limits of its approaches, and perhaps provide means to try and overcome them.

\section{References}

[Adam 2000] $\quad$ S. Adam, J-M. Ogier, C. Cariou, R. Mullot, J. Labiche, J. Gardes: Symbol and character recognition: application to engineering drawings. IJDAR 3(2): 89-101 (2000) 


\begin{tabular}{|c|c|}
\hline $\begin{array}{l}\text { [Antoine } \\
\text { 1992] }\end{array}$ & $\begin{array}{l}\text { D. Antoine, S. Collin and K. Tombre. Analysis of Technical Documents: The REDRAW System. In } \\
\text { H.S. Baird, H. Bunke and K. Yamamoto, editors,Structured Document Image Analysis, pages 385- } \\
\text { 402. Springer Verlag, Berlin/Heidelberg, } 1992 \text {. }\end{array}$ \\
\hline [Arias 1995] & $\begin{array}{l}\text { J.F. Arias, C.P. Lai, S. Surya, R. Kasturi, A. Chhabra, Interpretation of Telephone System Manhole } \\
\text { Drawings, Pattern Recognition Letters, 16, pp 355-369, } 1995 .\end{array}$ \\
\hline $\begin{array}{l}\text { [Baum et al. } \\
\text { 1997] }\end{array}$ & $\begin{array}{l}\text { L. S. Baum, J. H. Boose, and R. J. Kelley: Graphics Recognition for a Large-Scale Airplane } \\
\text { Information System, GREC, Lecture Notes in Computer Science, Vol. } 1389 \text { Springer (1997), p. 291- } \\
301 .\end{array}$ \\
\hline [Boatto 1992] & $\begin{array}{l}\text { L. Boatto and al, An Interpretation System for Land Register Maps, IEEE Computer Magazine, 25(7), } \\
\text { pp 25-33, } 1992 .\end{array}$ \\
\hline $\begin{array}{l}\text { [Broelemann } \\
\text { 2012] }\end{array}$ & $\begin{array}{l}\text { Broelemann, K. \& Jiang, X.: “A region-based method for sketch map segmentation”. Post-Conference } \\
\text { Proceedings of the GREC 2011. Seoul. Korea. Springer (to appear 2012) }\end{array}$ \\
\hline $\begin{array}{l}\text { [Coüasnon } \\
\text { 2006] }\end{array}$ & $\begin{array}{l}\text { B. Coüasnon: DMOS, a generic document recognition method: application to table structure analysis } \\
\text { in a general and in a specific way. IJDAR 8(2-3): 111-122 (2006) }\end{array}$ \\
\hline $\begin{array}{l}\text { [Coustaty } \\
2012]\end{array}$ & $\begin{array}{l}\text { Coustaty Mickaël, Uttama Surapong, Ogier Jean-Marc, Extraction of Light and Specific Features for } \\
\text { Historical Image Indexing and Matching, ICPR } 2012 \text { - To appear }\end{array}$ \\
\hline [Das 1997] & $\begin{array}{l}\text { Atish K. Das, Noshir A. Langrana: Recognition and Integration of Dimension Sets in Vectorized } \\
\text { Engineering Drawings. Computer Vision and Image Understanding 68(1): 90-108 (1997) }\end{array}$ \\
\hline $\begin{array}{l}\text { [Den Hartog } \\
1996]\end{array}$ & $\begin{array}{l}\text { J.E. Den Hartog, T.K. ten Kate, J.J. Gerbrands, Knowledge-based interpretation of Utility Maps, } \\
\text { Computer Vision and Image Understanding, 63(1), pp 105-117, } 1996 .\end{array}$ \\
\hline $\begin{array}{l}\text { [Deseilligny } \\
1998]\end{array}$ & $\begin{array}{l}\text { M.P. Deseilligny, R. Mariani, J. Labiche, R. Mullot, Topographic Maps Automatic Interpretation : } \\
\text { Some proposed Strategies, Lecture Notes in Computer Science, K. Tombre and A.K. Chhabra } \\
\text { (editors), Vol. 70, } \mathrm{n}^{\circ} 3 \text {, pp. 438-451, } 1998 .\end{array}$ \\
\hline $\begin{array}{l}\text { [Devaux et al. } \\
\text { 1999] }\end{array}$ & $\begin{array}{l}\text { Pierre M. Devaux, Daniel B. Lysak, Rangachar Kasturi: A complete system for the intelligent } \\
\text { interpretation of engineering drawings. IJDAR 2(2-3): 120-131 (1999) }\end{array}$ \\
\hline [Dori 1999] & $\begin{array}{l}\text { Dov Dori, Liu Wenyin: Automated CAD conversion with the Machine Drawing Understanding } \\
\text { System: concepts, algorithms, and performance. IEEE Transactions on Systems, Man, and } \\
\text { Cybernetics, Part A 29(4): 411-416 (1999) }\end{array}$ \\
\hline $\begin{array}{l}\text { [Dosch et al. } \\
2000]\end{array}$ & $\begin{array}{l}\text { Philippe Dosch, Karl Tombre, Christian Ah-Soon, Gérald Masini: A complete system for the analysis } \\
\text { of architectural drawings. IJDAR 3(2): 102-116 (2000) }\end{array}$ \\
\hline \multicolumn{2}{|l|}{ [Grenier 2000] } \\
\hline [Janssen 1995] & $\begin{array}{l}\text { R. D.T. Janssen, The application of model-based image processing to the interpretation of maps, PhD } \\
\text { thesis, technische Universiteit, Delft, September } 1995\end{array}$ \\
\hline [Joseph 1992] & $\begin{array}{l}\text { S.H. Joseph, P. Pridmore, Knowledge-directed Interpretation of Line Drawing Images, IEEE Trans. on } \\
\text { PAMI, 14(9), pp 928-940, } 1992 .\end{array}$ \\
\hline [Kasturi 1990] & $\begin{array}{l}\text { R. Kasturi and al, A System for Interpretation of Line Drawing, IEEE Trans. on PAMI, 12(10), pp } \\
\text { 978-992, } 1990 .\end{array}$ \\
\hline [Kasturi 1996] & $\begin{array}{l}\text { R. Kasturi and K. Tombre (editors). Graphics Recognition - Methods and Applications, Lecture Notes } \\
\text { in Computer Science, vol. 1072, Springer Verlag, } 1996\end{array}$ \\
\hline $\begin{array}{l}\text { [Langrana } \\
1997]\end{array}$ & $\begin{array}{l}\text { Noshir A. Langrana, Yuan Chen, Atish K. Das: Feature Identification from Vectorized Mechanical } \\
\text { Drawings. Computer Vision and Image Understanding 68(2): 127-145 (1997) }\end{array}$ \\
\hline [Li 2012] & $\begin{array}{l}\text { Real Scene Graphics Symbol Recognition, Linlin Li and Chew Lim Tan } \\
\text { Post-Conference Proceedings of the GREC 2011. Seoul. Korea. Springer (to appear 2012) }\end{array}$ \\
\hline [Lu et al. & Tong Lu, Chiew-Lan Tai, Huafei Yang, Shijie Cai: A Novel Knowledge-Based System for \\
\hline
\end{tabular}




\begin{tabular}{|c|c|}
\hline 2009] & $\begin{array}{l}\text { Interpreting Complex Engineering Drawings: Theory, Representation, and Implementation. IEEE } \\
\text { Trans. Pattern Anal. Mach. Intell. 31(8): 1444-1457 (2009) }\end{array}$ \\
\hline [Mas 2010] & $\begin{array}{l}\text { Joan Mas, Josep Lladós, Gemma Sánchez, Joaquim Armando Pires Jorge: A syntactic approach based } \\
\text { on distortion-tolerant Adjacency Grammars and a spatial-directed parser to interpret sketched } \\
\text { diagrams. Pattern Recognition 43(12): 4148-4164 (2010) }\end{array}$ \\
\hline [Nagy 2004] & George Nagy: Visual Pattern Recognition in the Years Ahead. ICPR (4) 2004: 7-10 \\
\hline [Nguyen 2009] & $\begin{array}{l}\text { Thi-Oanh Nguyen, Salvatore Tabbone, Alain Boucher: A Symbol Spotting Approach Based } \\
\text { on the Vector Model and a Visual Vocabulary. ICDAR 2009: 708-712 }\end{array}$ \\
\hline [Ogier 1998] & $\begin{array}{l}\text { J.M. Ogier R. Mullot, J. Labiche, Y. Lecoutier, Methodology and evaluation of a document } \\
\text { interpretation device, in Proc. SCI'98 and ISAS'98, World Multiconference on Systemics, Cybernetics } \\
\text { and Informatics and the 4th International Conference on Information Systems Analysis and Synthesis, } \\
\text { Orlando, USA, Vol 2, pp 457-463, 12-16 Juillet, } 1998\end{array}$ \\
\hline [Ogier 2000-a] & $\begin{array}{l}\text { Jean-Marc Ogier,, De l'image au sens, Habilitation à diriger de recherches thesis, University of } \\
\text { Rouen, France }\end{array}$ \\
\hline [Ogier 2000-b] & $\begin{array}{l}\text { Jean-Marc Ogier, Rémy Mullot, Jacques Labiche, Yves Lecourtier: Semantic coherency: the basis of } \\
\text { an image interpretation device-application to the cadastral map interpretation. IEEE Transactions on } \\
\text { Systems, Man, and Cybernetics, Part B 30(2): 322-338 (2000) }\end{array}$ \\
\hline $\begin{array}{l}\text { Pasternak } \\
1994]\end{array}$ & $\begin{array}{l}\text { B. Pasternak, Processing Imprecise and Structural Distorted Line Drwings by an Adaptable Drawing } \\
\text { Interpretation Kernel, in Proc. of IAPR Workshop on Document Analysis Systems, Kaiserslautern, } \\
\text { Germany, pp 349-365, } 1994 .\end{array}$ \\
\hline [Ogier 2001] & $\begin{array}{l}\text { J.M. Ogier, R. Mullot, J. Labiche, Y. Lecourtier, Technical Map Interpretation: A Distributed } \\
\text { Approach, (PAA) International Journal of Pattern Analysis and Applications (2001)3:88-103, } \\
2001\end{array}$ \\
\hline $\begin{array}{l}\text { [Pasternak } \\
1995]\end{array}$ & $\begin{array}{l}\text { B. Pasternak, B. Neumann, The Role of Taxonomy in Drawing Interpretation, in Proc. 3rd } \\
\text { International Conference on Document Analysis and Recognition, Montreal, Canada, pp 799-802, } \\
1995 .\end{array}$ \\
\hline $\begin{array}{l}\text { [Raveaux } \\
2010]\end{array}$ & $\begin{array}{l}\text { R. Raveaux, Graph Mining and Graph Classification: Application to cadastral map analysis, PhD } \\
\text { thesis, University of la Rochelle, France, November } 2010\end{array}$ \\
\hline [Rendek 2006] & $\begin{array}{l}\text { J. Rendek, B. Lamiroy and K. Tombre, “A Few Steps Towards On-the-Fly Symbol Recognition with } \\
\text { Relevance Feed- back”, in 7th International Workshop, Document Analysis and Systems, Nelson, } \\
\text { New Zealand, Springer-Verlag, Lecture Notes in Computer Science, Volume 3872, January 2006, pp. } \\
\text { 604-615. }\end{array}$ \\
\hline [Rusiñol 2010] & $\begin{array}{l}\text { Marçal Rusiñol, Agnés Borràs, Josep Lladós, "Relational indexing of vectorial primitives for symbol } \\
\text { spotting in line-drawing images", Pattern Recognition Letters } 31 \text { (2010) 188-201 }\end{array}$ \\
\hline $\begin{array}{l}\text { [Rosenberger } \\
1998]\end{array}$ & $\begin{array}{l}\text { C. Rosenberger C., Ogier J-M., C. Cariou, Chehdi K. , "A statistical Solution to Evaluate Image } \\
\text { Processing Techniques ", 7th international conference IPMU, Vol } 2 \text { pp 1648-1645, PARIS. Juillet } \\
1998\end{array}$ \\
\hline [Samet 1994] & $\begin{array}{l}\text { Hanan Samet, Aya Soffer, A Legend-Driven Geographic Symbol Recognition System } \\
\text { Proceedings of the 12th International Conference on Pattern Recognition, volume II (1994) }\end{array}$ \\
\hline $\begin{array}{l}\text { [Shimada } \\
\text { 1995] }\end{array}$ & $\begin{array}{l}\text { S. Shimada, K. Maruyama ; A. Matsumoto, K. Hiraki, Agent-Based Parallel Recognition Method of } \\
\text { Contour Lines, in Proc. ICDAR'95, Montreal, Canada, pp 154-157, } 1995\end{array}$ \\
\hline $\begin{array}{l}\text { [Shimotsuji } \\
1992]\end{array}$ & $\begin{array}{l}\text { Shigeyoshi Shimotsuji, Osamu Hori, Mieko Asano, Kaoru Suzuki, Fumihiko Hoshino, Toshiaki Ishii: } \\
\text { A Robust Recognition System for a Drawing Superimposed on a Map. IEEE Computer 25(7): 56-59 } \\
\text { (1992) }\end{array}$ \\
\hline [Smeulders & Arnold W. M. Smeulders, Carlo de Boer: Design and Performance in Object Recognition. GREC \\
\hline
\end{tabular}




\begin{tabular}{|l|l|}
\hline 1998$]$ & 1997: 335-346 \\
\hline [Suzuki 1990] & $\begin{array}{l}\text { Satoshi Suzuki, Toyomichi Yamada: MARIS: map recognition input system. Pattern Recognition } \\
\text { 23(8): 919-933 (1990) }\end{array}$ \\
\hline $\begin{array}{l}\text { [Thi 2011 } \\
\text { 1993] }\end{array}$ & $\begin{array}{l}\text { Thi Thuong Huyen Nguyen, Mickaël Coustaty, Jean-Marc Ogier: Bags of Strokes Based Approach for } \\
\text { Classification and Indexing of Drop Caps. ICDAR 2011: 349-353 }\end{array}$ \\
\hline [Tombre 2003] & K. Tombre, B. Lamiroy: Graphics Recognition - from Re-engineering to Retrieval. ICDAR 2003 \\
\hline $\begin{array}{l}\text { [Vaxivière } \\
\text { 1995] }\end{array}$ & $\begin{array}{l}\text { P. Vaxivière, K. Tombre, Knowledge Organization and Interpretation Process in Engineering Drawing } \\
\text { Interpretation, in A.L. Spitz and A. Dengel, eds, Document Analysis Systems, pp 307-317, World }\end{array}$ \\
\hline Scientific, 1995.
\end{tabular}

